\title{
Directional recurrence and directional rigidity for infinite measure preserving actions of nilpotent lattices
}

\author{
ALEXANDRE I. DANILENKO \\ Institute for Low Temperature Physics \& Engineering of National Academy of \\ Sciences of Ukraine, 47 Lenin Avenue, Kharkov 61164, Ukraine \\ (e-mail: alexandre.danilenko@gmail.com)
}

(Received 15 October 2014 and accepted in revised form 16 October 2015)

\begin{abstract}
Let $\Gamma$ be a lattice in a simply connected nilpotent Lie group $G$. Given an infinite measure-preserving action $T$ of $\Gamma$ and a 'direction' in $G$ (i.e. an element $\theta$ of the projective space $P(\mathfrak{g})$ of the Lie algebra $\mathfrak{g}$ of $G)$, some notions of recurrence and rigidity for $T$ along $\theta$ are introduced. It is shown that the set of recurrent directions $\mathcal{R}(T)$ and the set of rigid directions for $T$ are both $G_{\delta}$. In the case where $G=\mathbb{R}^{d}$ and $\Gamma=\mathbb{Z}^{d}$, we prove that (a) for each $G_{\delta}$-subset $\Delta$ of $P(\mathfrak{g})$ and a countable subset $D \subset \Delta$, there is a rank-one action $T$ such that $D \subset \mathcal{R}(T) \subset \Delta$ and (b) $\mathcal{R}(T)=P(\mathfrak{g})$ for a generic infinite measure-preserving action $T$ of $\Gamma$. This partly answers a question from a recent paper by Johnson and Şahin. Some applications to the directional entropy of Poisson actions are discussed. In the case where $G$ is the Heisenberg group $H_{3}(\mathbb{R})$ and $\Gamma=H_{3}(\mathbb{Z})$, a rank-one $\Gamma$-action $T$ is constructed for which $\mathcal{R}(T)$ is not invariant under the natural 'adjoint' $G$-action.
\end{abstract}

\section{Introduction}

Subdynamics is the study of the relationship between the dynamical properties of the action of a group $G$, and those of the action restricted to subgroups of $G$. In this paper, we consider measure-preserving actions defined on $\sigma$-finite standard measure spaces. In the 1980s Milnor generalized the study of subdynamics by defining a concept of directional entropy of a $\mathbb{Z}^{d}$-action in every direction, including the irrational directions for which there is no associated subgroup action [Mi]. To this end, he considered $\mathbb{Z}^{d}$ as a lattice in $\mathbb{R}^{d}$ and he exploited the geometry of mutual position of this lattice and the one-dimensional subspaces (i.e. directions) in $\mathbb{R}^{d}$. For a detailed account on the directional entropy of $\mathbb{Z}^{2}$ actions and some applications to topological dynamics (expansive subdynamics) we refer to $[\mathbf{P a}]$ and references therein. In a recent paper [JoSa], Johnson and Şahin applied the 'directional approach' to study recurrence properties of infinite measure-preserving $\mathbb{Z}^{2}$ - 
actions. They were motivated by Feldman's proof of the ratio ergodic theorem [Fel]. In particular, they showed that, for each such an action, say, $T$, the set $\mathcal{R}(T)$ of all recurrent directions of $T$ is a $G_{\delta}$-subset of the circle $\mathbb{T}$. They also exhibited examples of rank-one actions $T$ and $T^{\prime}$ with $R(T)=\emptyset$ and $\mathbb{T} \neq \mathcal{R}\left(T^{\prime}\right) \supset\left\{e^{\pi i q} \mid q \in \mathbb{Q}\right\}$. They raised a question: which $G_{\delta}$-subsets of $\mathbb{T}$ are realizable as recurrence sets, that is, appear as $R(T)$ for some $T$ ? We answer this question in part.

- We show that each countable $G_{\delta}$ is a recurrence set.

- $\quad$ More generally, for each $G_{\delta}$-subset $\Delta$ of the projective space $P\left(\mathbb{R}^{d}\right)$ and a countable subset $D$ of $\Delta$, there is a rank-one infinite measure-preserving free $\mathbb{Z}^{d}$-action $T$ such that $D \subset \mathcal{R}(T) \subset \Delta$ (Theorem 5.2).

- We also prove that a generic infinite measure-preserving action $T$ of $\mathbb{R}^{d}$ is recurrent in every direction: i.e. $\mathcal{R}(T)=P\left(\mathbb{R}^{d}\right)$ (Theorem 6.6).

In parallel to this, we introduce a concept of directional rigidity for $\mathbb{Z}^{d}$-actions and obtain similar results for realization of $G_{\delta}$-subsets of $P\left(\mathbb{R}^{d}\right)$ as rigidity sets.

As a by product, we obtain some examples of Poisson $\mathbb{R}^{d}$-actions with the following entropy properties.

- There is a Poisson action $V=\left(V_{g}\right)_{g \in \mathbb{R}^{d}}$ of 0 entropy such that, for each non-zero $g \in \mathbb{R}^{d}$, the transformation $V_{g}$ is Bernoulli of infinite entropy (Proposition 6.7).

- $\quad$ For each $G_{\delta}$-subset $\Delta \subset P\left(\mathbb{R}^{d}\right)$ and a countable subset $D$ of $\Delta$, there is a Poisson action $V=\left(V_{g}\right)_{g \in \mathbb{R}^{d}}$ of zero entropy such that, for each non-zero $g \notin \bigcup_{\theta \in \Delta} \theta$, the transformation $V_{g}$ is Bernoulli of infinite entropy and, for each $g \in \bigcup_{\theta \in D} \theta$, the transformation $V_{g}$ is rigid and hence of zero entropy (Proposition 6.8).

In this connection we recall the main result from [FeKa]: there is a Gaussian action $V=$ $\left(V_{g}\right)_{g \in \mathbb{Z}^{2}}$ of zero entropy such that every transformation $V_{g}, 0 \neq g \in \mathbb{Z}^{2}$, is Bernoulli.

We extend the concepts of directional recurrence and directional rigidity to actions of lattices $\Gamma$ in simply connected nilpotent Lie groups $G$. By a 'direction' we now mean a one-parameter subgroup in $G$. Thus the set of all directions is the projective space $P(\mathfrak{g})$, where $\mathfrak{g}$ denotes the Lie algebra of $G$. As in the Abelian case (considered originally in $[\mathbf{J o S a}]$ ), we show the following.

- $\quad$ Given a measure-preserving action $T$ of $\Gamma$, the set $\mathcal{R}(T)$ of all recurrent directions of $T$ is a $G_{\delta}$ in $P(\mathfrak{g})$ (Theorems 3.5 and 3.6).

Since $G$ acts on $P(\mathfrak{g})$ via the adjoint representation, we define another invariant $\mathcal{E} \mathcal{R}(T)$ of even recurrence for $T$ as the largest $G$-invariant subset of $\mathcal{R}(T)$.

- Some examples of rank-one actions $T$ of the Heisenberg group $H_{3}(\mathbb{Z})$ are constructed for which $\mathcal{R}(T)$ is either empty (Theorem 7.1), countably infinite (Theorem 7.2) or uncountable (Theorem 7.3) $\dagger$.

- $\quad$ An example of $T$ is given such that $\mathcal{E R}(T) \neq \mathcal{R}(T)$ (Theorem 7.2).

Given an action $T$ of $\Gamma$, we can define a natural analog of the 'suspension flow' corresponding to $T$. This is the induced (in the sense of Mackey) action $\widetilde{T}$ of $G$ associated with $T$. Since $\mathcal{R}(T)$ coincides with the set $\mathcal{R}(\widetilde{T})$ of conservative $\mathbb{R}$-subactions of $\widetilde{T}$ in the Abelian case [JoSa], it is natural to conjecture that $\mathcal{E} \mathcal{R}(T)=\mathcal{R}(\widetilde{T})$ in the general case. It remains an open problem. However, the analogous claim for the rigidity sets does not hold in the non-Abelian case (Remark 3.2).

$\dagger$ We consider $H_{3}(\mathbb{Z})$ as a lattice in the three-dimensional real Heisenberg group $H_{3}(\mathbb{R})$. 
The outline of the paper is as follows. In $\S 2$, we introduce the main concepts and invariants related to the directional recurrence and rigidity. In $\S 3$, we discuss relationship between the directional recurrence and rigidity of an action of a lattice in a nilpotent Lie group and similar properties of the suspension flow, i.e. the induced action of the underlying Lie group. It is also shown here that the sets of recurrent and rigid directions are both $G_{\delta}$. In $\S 4$, we recall the $(C, F)$-construction of rank-one actions and provide a sufficient condition for directions to be recurrent in terms of the $(C, F)$-parameters. This condition is used in $\S 5$ to construct rank-one actions of $\mathbb{Z}^{d}$ with various sets of recurrent directions. In $\S 6$, we prove that a generic $\mathbb{Z}^{d}$-action is recurrent in every direction. This section also contains some applications to the directional entropy of Poisson actions. In $\S 7$, we study directional recurrence of infinite measure-preserving actions of $H_{3}(\mathbb{Z})$. The final $\S 8$ contains a list of open problems and concluding remarks.

\section{Recurrence, even recurrence, rigidity and even rigidity along directions}

Let $G$ be a simply connected nilpotent Lie group, $\mathfrak{g}$ the Lie algebra of $G$ and $\exp : \mathfrak{g} \rightarrow G$ the exponential map. We note that exp is a diffeomorphism of $\mathfrak{g}$ onto $G$ [Mal]. Let $P(\mathfrak{g})$ denote the set of lines (i.e. one-dimensional subspaces) in $\mathfrak{g}$. We endow $P(\mathfrak{g})$ with the usual topology of projective space. Then $P(\mathfrak{g})$ is a compact manifold. The adjoint $G$ action on $\mathfrak{g}$ induces a smooth $G$-action on $P(\mathfrak{g})$. We denote this action by the symbol $\because$ Given $v \in \mathfrak{g} \backslash\{0\}$, we let $\exp (v):=\{\exp (t v) \mid t \in \mathbb{R}\}$. Then $\exp (v)$ is a closed onedimensional subgroup of $G$. We note that if $w=t v$ for some $t \in \mathbb{R} \backslash\{0\}$, then $\exp (w)=$ $\exp (v)$. Hence, for each line $\theta \in P(\mathfrak{g})$, the notation $\exp (\theta)$ is well defined. Moreover, $g \exp (\theta) g^{-1}=\exp (g \cdot \theta)$ for each $g \in G$.

Let $R=\left(R_{g}\right)_{g \in G}$ be a measure-preserving action of $G$ on a $\sigma$-finite standard measure space $(Y, \mathfrak{Y}, v)$.

\section{Definition 2.1.}

(i) We recall that $R$ is called conservative if for each subset $B \in \mathfrak{Y}, v(B)>0$, and for each compact set $K \subset G$, there is an element $g \in G \backslash K$, such that

$$
v\left(B \cap R_{g} B\right)>0 .
$$

(ii) We call $R$ recurrent along a line $\theta \in P(\mathfrak{g})$ if the flow $(\exp (t v))_{t \in \mathbb{R}}$ is conservative for some (and hence for each) $v \in \theta \backslash\{0\}$.

(iii) We recall that $R$ is called rigid if there is a sequence $\left(g_{n}\right)_{n \geq 1}$ of elements in $G$ such that $g_{n} \rightarrow \infty$ and

$$
\lim _{n \rightarrow \infty} v\left(B \cap R_{g_{n}} B\right)=\mu(B)
$$

for each subset $B \in \mathfrak{Y}$ of finite measure.

(iv) We call $R$ rigid along a line $\theta \in P(\mathfrak{g})$ if the flow $(\exp (t v))_{t \in \mathbb{R}}$ is rigid for some (and hence for each) $v \in \theta \backslash\{0\}$.

Denote by $\mathcal{R}(R)$ the set of all $\theta \in P(\mathfrak{g})$ such that $R$ is recurrent along $\theta$. Denote by $\mathcal{R} i(R)$ the set of all $\theta \in P(\mathfrak{g})$ such that $R$ is rigid along $\theta$. Of course, $\mathcal{R} i(R) \subset \mathcal{R}(R)$. It is easy to see that if a $G$-action $R^{\prime}$ is isomorphic to $R$ then $\mathcal{R}\left(R^{\prime}\right)=\mathcal{R}(R)$ and $\mathcal{R} i\left(R^{\prime}\right)=$ $\mathcal{R} i(R)$. 
Proposition 2.2. The sets $\mathcal{R}(R)$ and $\mathcal{R} i(R)$ are $G$-invariant.

Proof. Let $\theta \in \mathcal{R}(R)$. Fix an element $g_{0} \in G$. Take a subset $B \subset Y$ of positive measure and a compact $K \subset G$. Since $R$ is recurrent along $\theta$, there is $g \in \exp (\theta)$ such that $g \notin K$ such that $v\left(B \cap R_{g} B\right)>0$. Hence

$$
0<v\left(R_{g_{0}} B \cap R_{g_{0}} R_{g} B\right)=v\left(R_{g_{0}} B \cap R_{g_{0} g_{g_{0}}^{-1}} R_{g_{0}} B\right) .
$$

Since $g_{0} g g_{0}^{-1} \in \exp \left(g_{0} \cdot \theta\right)$ and $g_{0} g g_{0}^{-1} \notin g_{0} K g_{0}^{-1}$, it follows that the flow $\left(R_{g}\right)_{g \in g_{0} \cdot \theta}$ is conservative. Thus $\mathcal{R}(R)$ is $G$-invariant. In a similar way we can verify that $\mathcal{R} i(R)$ is $G$-invariant.

From now on we fix a lattice $\Gamma$ in $G$. We recall that there exists a lattice in $G$ if and only if the structural constants of $\mathfrak{g}$ are all rational [Mal]. Moreover, every lattice in $G$ is uniform [Mal], i.e. co-compact. We fix a right-invariant metric $\operatorname{dist}(\cdot, \cdot)$ on $G$, compatible with the topology.

Let $T=\left(T_{\gamma}\right)_{\gamma \in \Gamma}$ be a measure-preserving action of $\Gamma$ on a $\sigma$-finite standard measure space $(X, \mathfrak{B}, \mu)$. Although, in general, $T$ does not extend to a $G$-action on $(X, \mathfrak{B}, \mu)$, it is possible to give an analog of Definition 2.1 for $T$.

\section{Definition 2.3.}

(i) We call $T$ recurrent along a line $\theta \in P(\mathfrak{g})$ if, for each $\epsilon>0$ and every subset $A \in$ $\mathfrak{B}, \mu(A)>0$, there is an element $\gamma \in \Gamma \backslash\left\{1_{\Gamma}\right\}$ and an element $g \in \exp (\theta)$ such that $\operatorname{dist}(\gamma, g)<\epsilon$ and $\mu\left(A \cap T_{\gamma} A\right)>0$.

(ii) We call $T$ evenly recurrent along a line $\theta \in P(\mathfrak{g})$ if $T$ is recurrent along every line from the $G$-orbit of $\theta$.

(iii) We call $T$ rigid along a line $\theta \in P(\mathfrak{g})$ if there is a sequence $\left(\gamma_{n}\right)_{n \geq 1}$ of elements in $\Gamma$ such that $\lim _{n \rightarrow \infty} \inf _{g \in \exp (\theta)} \operatorname{dist}\left(\gamma_{n}, g\right)=0$ and

$$
\lim _{n \rightarrow \infty} \mu\left(A \cap T_{\gamma_{n}} A\right)=\mu(A)
$$

for each subset $A \in \mathfrak{B}$ with $\mu(A)<\infty \dagger$.

(iv) We call $T$ evenly rigid along a line $\theta \in P(\mathfrak{g})$ if $T$ is rigid along every line from the $G$-orbit of $\theta$.

We denote by $\mathcal{R}(T)$ the set of all $\theta \in P(\mathfrak{g})$ such that $T$ is recurrent along $\theta$. We denote by $\mathcal{R} i(T)$ the set of all $\theta \in P(\mathfrak{g})$ such that $T$ is rigid along $\theta$. In a similar way, we denote by $\mathcal{E} \mathcal{R}(T)$ and $\mathcal{E} \mathcal{R} i(T)$ the set of all $\theta \in P(\mathfrak{g})$ such that $T$ is evenly recurrent along them and evenly rigid along them, respectively.

Of course, $\mathcal{R}(T) \supset \mathcal{E R}(T), \mathcal{R} i(T) \supset \mathcal{E} \mathcal{R} i(T), \mathcal{R}(T) \supset \mathcal{R} i(T)$ and $\mathcal{E R}(T) \supset \mathcal{E} \mathcal{R} i(T)$. For $G$ Abelian, $\mathcal{R}(T)=\mathcal{E} \mathcal{R}(T)$ and $\mathcal{R} i(T)=\mathcal{E} \mathcal{R} i(T)$. However, in general, $\mathcal{R}(T) \neq$ $\mathcal{E R}(T)$ (see Theorem 7.2 below) and $\mathcal{R} i(T) \neq \mathcal{E} \mathcal{R} i(T)$.

$\dagger$ This means that $T_{\gamma_{n}} \rightarrow$ Id as $n \rightarrow \infty$ in the weak topology on the group of all $\mu$-preserving invertible transformations of $X$. 
Remark 2.4.

(i) It is easy to see that if $\theta$ is 'rational', i.e. the intersection $\Gamma \cap \exp (\theta)$ is non-trivial, say, there is $\gamma_{0} \neq 1_{\Gamma}$ such that $\Gamma \cap \exp (\theta)=\left\{\gamma_{0}^{n} \mid n \in \mathbb{Z}\right\}$, then $\theta$ is recurrent if and only if $\gamma_{0}$ (i.e. the action of $\mathbb{Z}$ generated by $\gamma_{0}$ ) is conservative. In a similar way, if $\theta$ is rigid if and only if $\gamma_{0}$ is rigid.

(ii) If $\theta \in \mathcal{R}(T)$, then we have $\{\gamma \cdot \theta \mid \gamma \in \Gamma\} \subset \mathcal{R}(T)$. In a similar way, if $\theta \in \mathcal{R} i(T)$, then we have $\{\gamma \cdot \theta \mid \gamma \in \Gamma\} \subset \mathcal{R} i(T)$. This can be shown in a similar way as in Proposition 2.2 (plus the fact that dist is right-invariant).

Given $g \in G$ and $\theta \in P(\mathfrak{g})$, we denote by $\operatorname{dist}(g, \exp (\theta))$ the distance from $g$ to the closed subgroup $\exp (\theta)$, i.e.

$$
\operatorname{dist}(g, \exp (\theta)):=\inf _{h \in \exp (\theta)} \operatorname{dist}(g, h)=\min _{h \in \exp (\theta)} \operatorname{dist}(g, h) .
$$

Since, in Definition 2.3(i), there is no estimate (from below) for the ratio $\mu$ ( $A \cap$ $\left.T_{\gamma} A\right) / \mu(A)$, the following lemma-which is equivalent to Definition 2.3(i)—is more useful for applications.

Lemma 2.5. Let $\theta \in \mathcal{R}(T)$. Then, for each $\epsilon>0$, a compact $K \subset G$ and a subset $A \subset X$ of finite measure, there is a Borel subset $A_{0} \subset A$ and Borel one-to-one map $R: A_{0} \rightarrow A$ and a Borel map $\vartheta: A_{0} \ni x \mapsto \vartheta_{x} \in \Gamma \backslash K$ such that $\mu\left(A_{0}\right) \geq 0.5 \mu(A)$ and $R x=T_{\vartheta_{x}} x$ and $\operatorname{dist}\left(\vartheta_{x}, \exp (\theta)\right)<\epsilon$ for all $x \in A_{0}$.

Proof. We use a standard exhaustion argument. Let

$$
\Gamma_{\epsilon}:=\{\gamma \in \Gamma \backslash\{1\} \mid \operatorname{dist}(\gamma, \exp (\theta))<\epsilon\} .
$$

Enumerate the elements of $\Gamma_{\epsilon}$ : i.e. let $\Gamma_{\epsilon}=\left\{\gamma_{n}\right\}_{n \geq 1}$. We now set $A_{1}:=A \cap T_{\gamma_{1}}^{-1} A, B_{1}:=$ $T_{\gamma_{1}} A_{1}, A_{2}:=\left(A \backslash\left(A_{1} \cup B_{1}\right)\right) \cap T_{\gamma_{2}}^{-1}\left(A \backslash\left(A_{1} \cup B_{1}\right)\right), B_{2}:=T_{\gamma_{2}} A_{2}$, and so on. Then we obtain two sequences $\left(A_{n}\right)_{n \geq 1}$ and $\left(B_{n}\right)_{n \geq 1}$ of Borel subsets of $A$ such that $A_{i} \cap A_{j}=$ $B_{i} \cap B_{j}=\emptyset$ whenever $i \neq j$ and $T_{\gamma_{i}} A_{i}=B_{i}$ for all $i$. We let $A_{0}:=\bigsqcup_{i \geq 1} A_{i}$ and $B_{0}:=$ $\bigsqcup_{i \geq 1} B_{i}$. It follows, from Definition 2.3(i), that $\mu\left(A \backslash\left(A_{0} \cup B_{0}\right)\right)=0$. Since $\mu\left(A_{0}\right)=$ $\mu\left(B_{0}\right)$, it follows that $\mu\left(A_{0}\right) \geq 0.5 \mu(A)$. It remains to let $\vartheta_{x}:=\gamma_{i}$ for all $x \in A_{i}, i \geq 1$.

\section{Recurrence and rigidity along directions in terms of the induced G-actions}

Denote by $\widetilde{T}=\left(\widetilde{T}_{g}\right)_{g \in G}$ the action of $G$ induced from $T$ (see [Ma, $\left.\mathbf{Z i}\right]$ ). We recall that the space of $\widetilde{T}$ is the product space $(G / \Gamma \times X, \lambda \times \mu)$, where $\lambda$ is the unique $G$-invariant probability measure on the homogeneous space $G / \Gamma$. To define $\widetilde{T}$ we first choose a Borel cross section $s: G / \Gamma \rightarrow G$ of the natural projection $G \rightarrow G / \Gamma$. Moreover, we may assume without loss of generality that $s(\Gamma)=1_{G}$ and $s$ is a homeomorphism when restricted to an open neighborhood of $\Gamma$, this neighborhood is of full measure and the measure of the boundary of the neighborhood is zero. Define a Borel map $h_{s}: G \times G / \Gamma \rightarrow \Gamma$ by setting

$$
h_{s}\left(g, g_{1} \Gamma\right)=s\left(g g_{1} \Gamma\right)^{-1} g s\left(g_{1} \Gamma\right) .
$$

Then $h_{s}$ satisfies the one-cocycle identity: i.e. $h_{s}\left(g_{2}, g_{1} g \Gamma\right) h_{s}\left(g_{1}, g \Gamma\right)=h_{s}\left(g_{2} g_{1}, g \Gamma\right)$ for all $g_{1}, g_{2}, g \in \Gamma$. We now set, for $g, g_{1} \in G$ and $x \in X$,

$$
\widetilde{T}_{g}\left(g_{1} \Gamma, x\right):=\left(g g_{1} \Gamma, T_{h_{s}\left(g, g_{1} \Gamma\right)} x\right) .
$$


Then $\left(\widetilde{T}_{g}\right)_{g \in G}$ is a measure-preserving action of $G$ on $(G / \Gamma \times X, \lambda \times \mu)$. We note that the isomorphism class of $\widetilde{T}$ does not depend on the choice of $s$.

THEOREM 3.1. Let $G=\mathbb{R}^{d}$ and $\Gamma=\mathbb{Z}^{d}, d \geq 1$. Then $\mathcal{R}(\widetilde{T})=\mathcal{R}(T)$ and $\operatorname{Ri}(\widetilde{T})=$ $\mathcal{R} i(T)$.

Proof. We consider the quotient space $G / \Gamma$ as $[0,1)^{d}$. Given $g=\left(g_{1}, \ldots, g_{d}\right) \in \mathbb{R}^{d}$, we let $[g]=\left(E\left(g_{1}\right), \ldots, E\left(g_{d}\right)\right)$ and $\{g\}:=\left(F\left(g_{1}\right), \ldots, F\left(g_{d}\right)\right)$, where $E($.$) and F($. denote the integer part and the fractional part of a real. If the cross section $s:[0,1)^{d} \rightarrow \mathbb{R}^{d}$ is given by the formula $s(y):=y$, then we have $h_{s}(g, y)=[g+y]$ for all $g \in G$ and $y \in[0,1)^{d}$.

(A) We first show that $\mathcal{R} i(T)=\mathcal{R} i(\widetilde{T})$. Let $\theta \in \mathcal{R} i(T)$. Then there are $\gamma_{n} \in \Gamma$ and $t_{n} \in \theta$ such that $\operatorname{dist}\left(\gamma_{n}, t_{n}\right) \rightarrow 0$ and $T_{\gamma_{n}} \rightarrow \operatorname{Id}_{X}$ weakly as $n \rightarrow \infty$. We claim that $\widetilde{T}_{t_{n}} \rightarrow$ $\operatorname{Id}_{(G / \Gamma) \times X}$ weakly as $n \rightarrow \infty$. Indeed, let $\epsilon_{n}:=t_{n}-\gamma_{n}$. Then

$$
\widetilde{T}_{t_{n}}(y, x)=\left(\left\{t_{n}+y\right\}, T_{\left[t_{n}+y\right]} x\right)=\left(\left\{\epsilon_{n}+y\right\}, T_{\gamma_{n}} T_{\left[\epsilon_{n}+y\right]} x\right) .
$$

Since the Lebesgue measure of the subset $Y_{n}:=\left\{y \in[0,1)^{d} \mid \epsilon_{n}+y \in[0,1)^{d}\right\}$ goes to one as $n \rightarrow \infty$ and $\left\{\epsilon_{n}+y\right\}=y$ and $\left[\epsilon_{n}+y\right]=0$ for all $y \in Y_{n}$, it follows that $\widetilde{T}_{t_{n}} \rightarrow$ $\operatorname{Id}_{(G / \Gamma) \times X}$ as $n \rightarrow \infty$. Thus we obtain that $\theta \in \mathcal{R} i(\widetilde{T})$.

Conversely, let $\theta \in \mathcal{R} i(\widetilde{T})$. Then there are $t_{n} \in \theta, n \in \mathbb{N}$, such that

$$
\widetilde{T}_{t_{n}} \rightarrow \operatorname{Id}_{(G / \Gamma) \times X} \text { weakly as } n \rightarrow \infty .
$$

It follows from (3.1) that the sequence of transformations $y \mapsto\left\{t_{n}+y\right\}$ of $G / \Gamma$ converge to $\operatorname{Id}_{G / \Gamma}$ as $n \rightarrow \infty$. This, in turn, implies that there is a sequence $\left(\gamma_{n}\right)_{n \in \mathbb{N}}$ of elements of $\Gamma$ such that $\lim _{n \rightarrow \infty} \operatorname{dist}\left(t_{n}, \gamma_{n}\right)=0$. Therefore, the Lebesgue measure of the subset $\left\{y \in G / \Gamma \mid\left[t_{n}+y\right]=\gamma_{n}\right\}$ converges to one as $n \rightarrow \infty$. Now (3.1) and (3.2) yield that $T_{\gamma_{n}} \rightarrow \operatorname{Id}_{X}$. Hence $\theta \in \mathcal{R} i(T)$.

(B) We now show that $\dagger \mathcal{R}(T)=\mathcal{R}(\widetilde{T})$. Take $\theta \in \mathcal{R}(T)$. Given a subset $A \subset G / \Gamma \times X$ of positive measure, a compact $K \subset G$ and $\epsilon>0$, we find two subsets $B \subset X$ and $C \subset$ $G / \Gamma$ of finite positive measure such that

$$
(\operatorname{Leb} \times \mu)(A \cap(B \times C))>0.99 \operatorname{Leb}(B) \mu(C) .
$$

For $t \in G$, we set $B_{t}:=\{y \in B \mid t+y \in B$ and $[t+y]=0\}$. Then we find $\epsilon_{1}>0$ so small that $\operatorname{Leb}\left(B_{t}\right)>0.5 \operatorname{Leb}(B)$ for each $t \in G$ such that $\operatorname{dist}(t, 0)<\epsilon_{1}$. By Lemma 2.5, there are elements $\gamma_{1}, \ldots, \gamma_{l} \in \Gamma, t_{1}, \ldots, t_{l} \in \theta \backslash K$ and pairwise disjoint subsets $C_{1}, \ldots, C_{l}$ of $C$ such that $\max _{1 \leq j \leq l} \operatorname{dist}\left(\gamma_{j}, t_{j}\right)<\min \left(\epsilon, \epsilon_{1}\right)$, the sets $T_{\gamma_{1}} A_{1}, \ldots, T_{\gamma_{l}} C_{l}$ are mutually disjoint subsets of $C$ and $\mu\left(\bigsqcup_{j=1}^{l} C_{j}\right)>0.4 \mu(C)$. We now let $A^{\prime}:=\bigsqcup_{j=1}^{l} B_{t_{j}} \times C_{j}$. Of course, $A^{\prime}$ is a subset of $B \times C$. We have

$$
\widetilde{T}_{t_{j}}(b, c)=\left(\left\{t_{j}+b\right\}, T_{\gamma_{j}} c\right) \subset B \times C \quad \text { if } b \in B_{j} \text { and } c \in C_{j}
$$

for each $j=1, \ldots, l$. Moreover, the sets $\widetilde{T}_{t_{j}}\left(B_{j} \times C_{j}\right), j=1, \ldots, l$, are pairwise disjoint and $(\operatorname{Leb} \times \mu)\left(\bigsqcup_{j=1}^{l}\left(B_{j} \times C_{j}\right)\right)>0.2(\operatorname{Leb} \times \mu)(B \times C)$. It now follows the proof of the inclusion $\mathcal{R}(T) \subset \mathcal{R}(\widetilde{T})$ was not completed there. 
from (3.3) that there is $j \in\{1, \ldots, l\}$ such that $($ Leb $\times \mu)\left(\widetilde{T}_{t_{j}}\left(A \cap\left(B_{j} \times C_{j}\right) \cap A\right)>0\right.$. Hence $\theta \in \mathcal{R}(\widetilde{T})$.

Conversely, let $\theta \in \mathcal{R}(\widetilde{T})$. Given $\epsilon>0$, let $Y=[1 / 2,1 / 2+\epsilon) \subset G / \Gamma$. It is easy to see that if $g Y \cap Y \neq \emptyset$ for some $g \in G$, then $\operatorname{dist}(g, \Gamma)<\epsilon$ and the map $Y \ni y \mapsto[g+y] \epsilon$ $\mathbb{Z}^{d}$ is constant. Let $A$ be a subset of $X$ of finite positive measure. Then there is $g \in \theta$ such that $\operatorname{dist}(g, 0)>100$ and

$$
0<(\operatorname{Leb} \times \mu)\left((Y \times A) \cap \widetilde{T}_{g}(Y \times A)\right)=\operatorname{Leb}(g Y \cap Y) \mu\left(A \cap T_{\gamma} A\right),
$$

where $\gamma:=[g+y] \in \Gamma$ for all $y \in Y$. It follows that $\operatorname{dist}(\gamma, \theta)<\epsilon$ and $\gamma \neq 0$. Hence $\theta \in \mathcal{R}(T)$.

Remark 3.2. We note that the equality $\mathcal{R} i(\widetilde{T})=\mathcal{E} \mathcal{R} i(T)$ does not hold for non-Abelian nilpotent groups. Consider, for instance, the case where $G=H_{3}(\mathbb{R})$ and $H=H_{3}(\mathbb{Z})$ (see $\$ 7$ for their definition). Let $T$ be an ergodic action of $H_{3}(\mathbb{Z})$. We claim that $\widetilde{T}$ is not rigid and hence $\operatorname{Ri}(\widetilde{T})=\emptyset$. Indeed, if $\widetilde{T}$ were rigid, then the quotient $G$-action by translations on $G / \Gamma$ is also rigid. However, the latter action is mixing relative to the subspace generated by all eigenfunctions [Au-Ha]. On the other hand, there are examples of weakly mixing $H_{3}(\mathbb{Z})$-actions $T$ such that $\mathcal{R} i(T)$ contains the line passing through the center [Da3].

COROllary 3.3. Let $G=\mathbb{R}^{d}$ and $\Gamma=\mathbb{Z}^{d}, d \geq 1$. If an action $T$ of $\Gamma$ is ergodic and extends to an action $\widehat{T}$ of $G$ on the same measure space where $T$ is defined, then $\mathcal{R}(T)=$ $\mathcal{R}(\widehat{T})$.

Proof. It follows, from the condition of the corollary, that the induced $G$-action $\widetilde{T}$ is isomorphic to the product $\widehat{T} \times D$, where $D$ is the natural $G$-action by translations on $G / \Gamma[\mathbf{Z i}$, Proposition 2.10]. Since $D$ is finite measure-preserving, $\mathcal{R}(\widehat{T} \times D)=\mathcal{R}(\widehat{T})$ (see Lemma 3.4(ii) below). It remains to apply Theorem 3.1.

We leave the proof of the following non-difficult statement to the reader as an exercise.

LEMMA 3.4. Let $F=\left(F_{t}\right)_{t \in \mathbb{R}}$ be a flow-preserving a $\sigma$-finite measure and let $S=\left(S_{t}\right)_{t \in \mathbb{R}}$ be a probability-preserving flow.

(i) $F$ is conservative if and only if the transformation $F_{1}$ is conservative.

(ii) $F$ is conservative if and only if the product flow $\left(F_{t} \times S_{t}\right)_{t \in \mathbb{R}}$ is conservative $\dagger$.

(iii) $F$ is rigid if and only if $F_{1}$ is rigid.

We now describe the 'topological type' of $\mathcal{R}(T)$ and $\mathcal{E R}(T)$ as subspaces of $P(\mathfrak{g})$. We first consider the Abelian case and provide a short proof of [JoSa, Theorem 1.3] stating that $\mathcal{R}(T)$ is a $G_{\delta}$.

THEOREM 3.5. Let $G=\mathbb{R}^{d}$ and $\Gamma=\mathbb{Z}^{d}, d \geq 1$. The subsets $\mathcal{R}(T)$ and $\mathcal{R} i(T)$ are both $G_{\delta}$ in $P\left(\mathbb{R}^{d}\right)$.

$\dagger$ A similar claim for transformations (i.e. $\mathbb{Z}$-actions) is proved in [Aa]. We note that (ii) follows from that claim and (i). 
Proof. Let $(\tilde{X}, \tilde{\mu})$ be the space of $\widetilde{T}$. Denote by $\operatorname{Aut}(\tilde{X}, \tilde{\mu})$ the group of all $\tilde{\mu}$-preserving invertible transformations of $\tilde{X}$. We endow it with the standard weak topology. Then $\operatorname{Aut}(\tilde{X}, \tilde{\mu})$ is a Polish group (see $[\mathbf{D a S i}]$ and references therein). Fix a norm on $\mathbb{R}^{d}$. Denote by $\mathcal{S}$ the unit ball in $\mathbb{R}^{d}$. We define a map $\mathfrak{m}: \mathcal{S} \rightarrow \operatorname{Aut}(\widetilde{X}, \tilde{\mu})$ by $\operatorname{setting} \mathfrak{m}(v):=\widetilde{T}_{v}$. It is obviously continuous. We recall that the subset $\mathfrak{R}$ of conservative infinite measurepreserving transformations of $(\tilde{X}, \tilde{\mu})$ is a $G_{\delta}$ in $\operatorname{Aut}(\tilde{X}, \tilde{\mu})[\mathbf{D a S i}]$. It follows from this fact and Lemma 3.4(i) that the set

$$
\mathfrak{m}^{-1}(\mathfrak{R})=\left\{v \in \mathcal{S} \mid \text { the flow }\left(\widetilde{T}_{t v}\right)_{t \in \mathbb{R}} \text { is conservative }\right\}
$$

is a $G_{\delta}$ in $\mathcal{S}$ : i.e. the intersection of a countable number of open subsets. Since $\mathfrak{m}^{-1}(\mathfrak{R})$ is centrally symmetric (i.e. if $v \in \mathfrak{m}^{-1}(\mathfrak{R})$, then $-v \in \mathfrak{m}^{-1}(\mathfrak{R})$ ), we may assume, without loss of generality, that these open sets are also centrally symmetric. The natural projection of $\mathcal{S}$ onto $P\left(\mathbb{R}^{d}\right)$ is just the 'gluing' of the pairs of centrally symmetric points. We note that the projection of $\mathfrak{m}^{-1}(\mathfrak{R})$ to $P\left(\mathbb{R}^{d}\right)$ is exactly $\mathcal{R}(\widetilde{T})$. It follows that $\mathcal{R}(\widetilde{T})$ is a $G_{\delta}$ in $P(\mathfrak{g})$. It remains to apply Theorem 3.1 .

To show that $\mathcal{R} i(T)$ is a $G_{\delta}$ argue in a similar way and use the fact that the set of rigid transformations is a $G_{\delta}$ in $\operatorname{Aut}(\tilde{X}, \tilde{\mu})[\mathbf{D a S i}]$ and apply Lemma 3.4(iii).

We now consider the general case (independently of Theorem 3.5).

THEOREM 3.6. The subsets $\mathcal{R}(T)$ and $\mathcal{R} i(T)$ are both $G_{\delta}$ in $P(\mathfrak{g})$.

Proof. Let $\Gamma \backslash\{1\}=\left\{\gamma_{k} \mid k \in \mathbb{N}\right\}$.

(A) We first prove that $\mathcal{R}(T)$ is a $G_{\delta}$. For each $g \in G$, the map

$$
P(\mathfrak{g}) \ni \theta \mapsto \operatorname{dist}(g, \exp (\theta)):=\inf _{h \in \exp (\theta)} \operatorname{dist}(g, h) \in \mathbb{R}
$$

is continuous. Now for a subset $A \subset X$ with $0<\mu(A)<\infty$ and $\epsilon>0$, we construct a sequence $A_{1}, A_{2}, \ldots$ of subsets in $A$ as given by (cf. with the proof of Lemma 2.5):

$$
\begin{gathered}
A_{1}:= \begin{cases}A \cap T_{\gamma_{1}}^{-1} A & \text { if } \operatorname{dist}\left(\gamma_{1}, \exp (\theta)\right)<\epsilon, \\
\emptyset & \text { otherwise, }\end{cases} \\
A_{2}:= \begin{cases}\left(A \backslash\left(A_{1} \cup T_{\gamma_{1}} A_{1}\right)\right) \cap T_{\gamma_{2}}^{-1}\left(A \backslash\left(A_{1} \cup T_{\gamma_{1}} A_{1}\right)\right) & \text { if } \operatorname{dist}\left(\gamma_{2}, \exp (\theta)\right)<\epsilon, \\
\varnothing & \text { otherwise, }\end{cases}
\end{gathered}
$$

and so on. Then (as in Lemma 2.5) $A_{i} \cap A_{j}=\emptyset, T_{\gamma_{i}} A_{i} \subset A$ and $T_{\gamma_{i}} A_{i} \cap T_{\gamma_{j}} A_{j}=\emptyset$ if $i \neq j$. For each $m \in \mathbb{N}$, we set

$$
\Theta_{\epsilon, A, m}:=\left\{\theta \in P(\mathfrak{g}) \mid \sum_{j \leq m} \mu\left(A_{j}\right)>0.4 \mu(A)\right\} .
$$

We note that for each $j>0$, the map $P(\mathfrak{g}) \ni \theta \mapsto \mu\left(A_{j}\right) \in \mathbb{R}$ is lower semicontinuous. Indeed, this map is (up to a multiplicative constant) the indicator function of the subset $\left\{\theta \mid \operatorname{dist}\left(\gamma_{j}, \exp (\theta)\right)<\epsilon\right\}$ which is open because (3.4) is continuous. It follows that $\Theta_{\epsilon, A, m}$ is an open subset in $P(\mathfrak{g})$. Fix a countable family $\mathfrak{D}$ of subsets of finite positive measure in $X$ such that $\mathfrak{D}$ is dense in $\mathfrak{B}$. We claim that

$$
\mathcal{R}(T)=\bigcap_{D \in \mathfrak{D}} \bigcap_{l=1}^{\infty} \bigcup_{m=1}^{\infty} \Theta_{1 / l, D, m} .
$$


Indeed, if $T$ is recurrent along a line $\theta \in P(\mathfrak{g})$, then, for each $\epsilon>0$ and each subset $A$ of positive measure, $\mu\left(\bigsqcup_{j} A_{j}\right) \geq 0.5 \mu(A)$ (as in Lemma 2.5). We then obtain that there exists $m>0$ with $\mu\left(\bigsqcup_{j=1}^{m} A_{i}\right)>0.4 \mu(A)$. Hence $\theta \in \Theta_{\epsilon, A, m}$. Now let $A$ run $\mathfrak{D}$ and let $\epsilon$ run $\{1 / l \mid l \in \mathbb{N}\}$. Then $\theta$ belongs to the right-hand side of (3.5).

Conversely, take $\theta$ from the right-hand side of (3.5). Let $A$ be a subset of $X$ of positive measure. Then there is $D \in \mathfrak{D}$ such that $\mu(A \cap D)>0.999 \mu(D)$. Take $l \in \mathbb{N}$. Select $m>0$ such that $\theta \in \Theta_{1 / l, D, m}$. Then

$$
\mu\left(\bigsqcup_{j \leq m} D_{j}\right)>0.4 \mu(D) \text { and hence } \mu\left(\bigsqcup_{j \leq m} T_{\gamma_{j}} D_{j}\right)>0.4 \mu(D) .
$$

Therefore there is $j<d$ with $\mu\left(T_{\gamma_{j}} A \cap A\right)>0$ and (because $\left.\theta \in \Theta_{1 / l, D, m}\right) \operatorname{dist}\left(\gamma_{j}\right.$, $\exp (\theta))<1 / m$.

(B) To show that $\operatorname{Ri}(T)$ is $G_{\delta}$ we first denote by $\tau$ a metric on $\operatorname{Aut}(X, \mu)$ compatible with the weak topology. Now it suffices to note that

$$
\mathcal{R} i(T)=\bigcap_{k=1}^{\infty} \bigcap_{N=1}^{\infty} \bigcup_{\left\{n>N \mid \tau\left(T_{\gamma_{n}}, \mathrm{Id}\right)<1 / k\right\}}\left\{\theta \in P(\mathfrak{g}) \mid \operatorname{dist}\left(\gamma_{n}, \exp (\theta)\right)<1 / k\right\}
$$

and use (3.4).

\section{4. $(C, F)$-construction and directional recurrence of rank-one actions}

We first recall a $(C, F)$-construction of group actions (see [Da1] for a detailed exposition and various applications).

Let $\left(C_{n}\right)_{n>0}$ and $\left(F_{n}\right)_{n \geq 0}$ be two sequences of finite subsets in $\Gamma$ such that the following conditions hold:

(I) $F_{0}=\{1\}, 1 \in C_{n}$ and $\# C_{n}>1$ for all $n$;

(II) $F_{n} C_{n+1} \subset F_{n+1}$ for all $n$;

(III) $F_{n} c \cap F_{n} c^{\prime}=\emptyset$ for all $c \neq c^{\prime} \in C_{n+1}$ and $n$; and

(IV) $\gamma F_{n} C_{n+1} C_{n+2} \cdots C_{m} \subset F_{m+1}$ eventually in $m$ for each $\gamma \in \Gamma$ and every $n$.

Then the infinite product space $X_{n}:=F_{n} \times C_{n+1} \times C_{n+1} \times \cdots$ is a (compact) Cantor set. It follows from (II) and (III) that the map

$$
X_{n} \ni\left(f_{n}, c_{n+1}, c_{n+2}, c_{n+3}, \ldots\right) \mapsto\left(f_{n} c_{n+1}, c_{n+2}, c_{n+3}, \ldots\right) \in X_{n+1}
$$

is a continuous embedding. Denote by $X$ the (topological) inductive limit of the sequence $X_{1} \subset X_{2} \subset \cdots$. Then $X$ is a locally compact Cantor set. For a subset $A \subset F_{n}$, we let $[A]_{n}:=\left\{x=\left(f_{n}, c_{n+1}, \ldots\right) \in X_{n} \mid f_{n} \in A\right\}$. Then $[A]_{n}$ is a compact-open subset of $X$. We call it an $n$-cylinder. The family of all cylinders (i.e. the family of all compactopen subsets of $X$ ) is a base of the topology in $X$. Given $\gamma \in \Gamma$ and $x \in X$, in view of (II) and (IV), there is $n$ such that $x=\left(f_{n}, c_{n+1}, \ldots\right) \in X_{n}$ and $\gamma f_{n} \in F_{n}$. Then we let $T_{\gamma} x:=\left(\gamma f_{n}, c_{n+1}, \ldots\right) \in X_{n} \subset X$. It is standard to verify that $T_{\gamma}$ is a well defined homeomorphism of $X$. Moreover, $T_{\gamma} T_{\gamma^{\prime}}=T_{\gamma \gamma^{\prime}}$ for all $\gamma, \gamma^{\prime} \in \Gamma$ : i.e. $T:=\left(T_{\gamma}\right)_{\gamma \in \Gamma}$ is a continuous action of $\Gamma$ on $X$. It is called the $(C, F)$-action of $\Gamma$ associated with $\left(C_{n}, F_{n-1}\right)_{n>0}$ (see [Da1, Da3]). This action is free and minimal. There is a unique (up to scaling) $T$-invariant $\sigma$-finite Borel measure $\mu$ on $X$. It is easy to compute that

$$
\mu\left([A]_{n}\right)=\frac{\# A}{\# C_{1} \cdots \# C_{n}}
$$


for all subsets $A \subset F_{n}, n>0$, provided that $\mu\left(X_{0}\right)=1$. We note that $\mu(X)=\infty$ if and only if

$$
\lim _{n \rightarrow \infty} \frac{\# F_{n}}{\# C_{1} \cdots \# C_{n}}=\infty .
$$

Of course, $(X, \mu, T)$ is an ergodic conservative dynamical system. It is of funny rank one (see [Da1] and [Da3] for the definition). Conversely, every funny rank-one free system appears this way: i.e. it is isomorphic to a $(C, F)$-system for an appropriately chosen sequence $\left(C_{n}, F_{n-1}\right)_{n \geq 1}$. We state, without proof, a lemma from [Da3].

LEMmA 4.1. Let $A$ be a finite subset $F_{n}$ and let $g \in G$. Then $[A]_{n} \cap T_{g}[A]_{n} \neq \emptyset$ if and only if $g \in \bigcup_{m>n} A C_{n+1} \cdots C_{m} C_{m}^{-1} \cdots C_{n+1}^{-1} A^{-1}$. Furthermore, if we let

$\mathcal{N}_{m}^{g, A}:=\left\{\left(a, c_{n+1}, \ldots, c_{m}\right) \in A \times C_{n+1} \times \cdots \times C_{m} \mid g a c_{n+1} \cdots c_{m} \in A C_{n+1} \cdots C_{m}\right\}$, then $\mu\left([A]_{n} \cap T_{g}[A]_{n}\right)=\lim _{m \rightarrow \infty}\left(\# \mathcal{N}_{m}^{g, A} / \# C_{1} \ldots \# C_{m}\right)$.

To state the next assertion we need more notation. Denote the natural projection by $\pi: \mathfrak{g} \backslash\{0\} \rightarrow P(\mathfrak{g})$. Let $\kappa$ be a metric on $P(\mathfrak{g})$ compatible with the topology. Given two sequences $\left(A_{n}\right)_{n=1}^{\infty}$ and $\left(B_{n}\right)_{n=1}^{\infty}$ of finite subsets in $G$, we write $A_{n} \gg B_{n}$ as $n \rightarrow \infty$ if

$$
\lim _{n \rightarrow \infty} \max _{a \in A_{n}, b \in B_{n}} \kappa(\pi(\log (a b), \pi(\log (a))=0 .
$$

Proposition 4.2. Let $T=\left(T_{\gamma}\right)_{\gamma \in \Gamma}$ be a $(C, F)$-action of $\Gamma$ associated with a sequence $\left(C_{n}, F_{n-1}\right)_{n=1}^{\infty}$ satisfying (I)-(IV). Then

(i) $\mathcal{R}(T) \subset \bigcap_{\gamma \in \Gamma} \gamma \cdot\left(\bigcap_{n=1}^{\infty} \overline{\bigcup_{m \geq n} \pi\left(\log \left(C_{n} \cdots C_{m} C_{m}^{-1} \cdots C_{n}^{-1} \backslash\{1\}\right)\right)}\right)$.

(ii) If, moreover, the group generated by all $C_{j}, j>0$, is commutative and $C_{j} \backslash\{1\} \gg$ $C_{1} \cdots C_{j-1}$ as $j \rightarrow \infty$, then

$$
\mathcal{R}(T) \subset \bigcap_{\gamma \in \Gamma} \gamma \cdot\left(\bigcap_{n=1}^{\infty} \overline{\bigcup_{m \geq n} \pi\left(\log \left(C_{m} C_{m}^{-1} \backslash\{1\}\right)\right)}\right) .
$$

(iii) If, in addition, there is $c_{j} \in \Gamma$ such that $C_{j}=\left\{1, c_{j}\right\}$ for each $j>0$, then

$$
\mathcal{R}(T) \subset \bigcap_{\gamma \in \Gamma} \gamma \cdot\left(\bigcap_{n=1}^{\infty} \overline{\left\{\pi\left(\log c_{m}\right) \mid m \geq n\right\}}\right) .
$$

Proof. (i) Let $\theta \in \mathcal{R}(T)$. Then for each $n>0$, there is a sequence $\left(\gamma_{m}\right)_{m=1}^{\infty}$ of elements of $\Gamma$ such that $\gamma_{m} \neq 1$ and $\mu\left(T_{\gamma_{m}}[1]_{n} \cap[1]_{n}\right)>0$ for each $m$ and $\operatorname{dist}\left(\gamma_{m}, \exp (\theta)\right) \rightarrow 0$ as $m \rightarrow \infty$. Hence we deduce from Lemma 4.1 that

$$
\inf \left\{\operatorname{dist}(\gamma, \exp (\theta)) \mid \gamma \in \bigcup_{m>n} C_{n+1} \cdots C_{m} C_{m}^{-1} \cdots C_{n+1}^{-1} \backslash\{1\}\right\}=0 .
$$

This yields that $\theta \in \overline{\pi\left(\log \left(\bigcup_{m>n} C_{n+1} \cdots C_{m} C_{m}^{-1} \cdots C_{n+1}^{-1} \backslash\{1\}\right)\right)}$. Therefore

$$
\mathcal{R}(T) \subset \bigcap_{n \geq 1} \overline{\bigcup_{m>n} \pi\left(\log \left(C_{n+1} \cdots C_{m} C_{m}^{-1} \cdots C_{n+1}^{-1} \backslash\{1\}\right)\right)} .
$$

Since $\mathcal{R}(T)$ is invariant under $\Gamma$, in view of Remark 2.4(ii), the claim (i) follows. 
(ii) Denote by $A$ the smallest closed Lie subgroup of $G$ containing all $C_{j}, j>0$. Since $A$ is Abelian, the restriction of $\log$ to $A$ is a group homomorphism. Hence the condition $C_{j} \backslash\{1\} \gg C_{1} \cdots C_{j-1} \quad$ as $j \rightarrow \infty$ implies $C_{j} C_{j}^{-1} \backslash\{1\} \gg C_{1} C_{1}^{-1} \cdots C_{j-1} C_{j-1}^{-1}$ as $j \rightarrow \infty$. Now (ii) easily follows from (i).

(iii) It suffices to note that $C_{m} C_{m}^{-1} \backslash\{1\}=\left\{c_{m}, c_{m}^{-1}\right\}$ and $\pi\left(\log c_{m}\right)=\pi\left(\log c_{m}^{-1}\right)$.

\section{Directional recurrence sets for actions of Abelian lattices}

In this section we consider the case of Abelian $G$ in more detail. Our purpose here is to realize various $G_{\delta}$-subsets of $P(\mathfrak{g})$ as $\mathcal{R}(T)$ for rank-one actions $T$ of $G$. Since $G$ is simply connected, there is $d>0$ such that $G=\mathbb{R}^{d}$. Hence $\mathfrak{g}=\mathbb{R}^{d}$ and the maps exp and $\log$ are the identities. Replacing $\Gamma$ with an automorphic lattice we may assume, without loss of generality, that $\Gamma=\mathbb{Z}^{d}$. In the subsequent work, we assume that $d>1$ (the case $d=1$ is trivial). By dist $(\cdot, \cdot)$ we denote the usual distance between a point and a closed subset of $\mathbb{R}^{d}$. We also note that $\mathcal{E} \mathcal{R}(T)=\mathcal{R}(T)$ for each measure-preserving action $T$ of $\Gamma$. We now restate Proposition 4.2 for the Abelian case.

Proposition 5.1. Let $T=\left(T_{\gamma}\right)_{\gamma \in \mathbb{Z}^{d}}$ be a $(C, F)$-action of $\mathbb{Z}^{d}$ associated with a sequence $\left(C_{n}, F_{n-1}\right)_{n=1}^{\infty}$ satisfying (I)-(IV). Then

(i) $\mathcal{R}(T) \subset \bigcap_{n=1}^{\infty} \overline{\pi\left(\sum_{j \geq n}\left(C_{j}-C_{j}\right) \backslash\{0\}\right)}$.

(ii) If, moreover, $C_{j} \backslash\{0\} \gg C_{1} \cup \cdots \cup C_{j-1}$ as $j \rightarrow \infty$, then

$$
\mathcal{R}(T) \subset \bigcap_{n=1}^{\infty} \overline{\bigcup_{m \geq n} \pi\left(\left(C_{m}-C_{m}\right) \backslash\{0\}\right) .}
$$

(iii) In, in addition, there is $c_{j} \in \mathbb{Z}^{d}$ such that $C_{j}=\left\{0, c_{j}\right\}$ for each $j>0$, then

$$
\mathcal{R}(T) \subset \bigcap_{n=1}^{\infty} \overline{\left\{\pi\left(c_{m}\right) \mid m \geq n\right\}} .
$$

The following two theorems are the main results of this section.

THEOREM 5.2. Let $\Delta$ be a $G_{\delta}$-subset of $P\left(\mathbb{R}^{d}\right)$ and let $D$ be a countable subset of $\Delta$. Then there is a rank-one free infinite measure-preserving action $T$ of $\mathbb{Z}^{d}$ such that $D \subset$ $\mathcal{R}(T) \subset \Delta$. In particular, each countable $G_{\delta}$-subset (e.g. each countable compact) of $P\left(\mathbb{R}^{d}\right)$ is realizable as $\mathcal{R}(T)$ for some rank-one free action $T$ of $\mathbb{Z}^{d}$.

Proof. First, suppose that $\Delta \neq \emptyset$. Then, without loss of generality, we may think that $D \neq \emptyset$. Let $\left(\delta_{n}\right)_{n=1}^{\infty}$ be a sequence such that $\delta_{n} \in D$, for each $n$, and every element of $D$ occurs in this sequence an infinite number of times. Let $\left(\epsilon_{n}\right)_{n=1}^{\infty}$ be a decreasing sequence of positive reals with $\lim _{n \rightarrow \infty} \epsilon_{n}=0$. There exists an increasing sequence $L_{1} \subset L_{2} \subset$ $\cdots$ of closed subsets in $P\left(\mathbb{R}^{d}\right)$ such that $P\left(\mathbb{R}^{d}\right) \backslash \Delta=\bigcup_{j \geq 1} L_{j}$. Let $L_{1}^{+} \subset L_{2}^{+} \subset \cdots$ be a sequence of open subsets in $P\left(\mathbb{R}^{d}\right)$ such that $L_{j}^{+} \supset L_{j}$ and $\delta_{j} \notin \overline{L_{j}^{+}}$, for each $j$, and $\bigcup_{j \geq 1} L_{j}^{+} \neq P\left(\mathbb{R}^{d}\right)$. We will construct, inductively, two sequences $\left(F_{n}\right)_{n=0}^{\infty}$ and $\left(C_{n}\right)_{n=1}^{\infty}$ satisfying (I)-(IV) and (4.1). We note, in advance, that, in our construction, $\# C_{n}=2$ and $F_{n}$ is a symmetric cube in $\mathbb{Z}^{d}$ : i.e. there is $a_{n} \in \mathbb{N}$ such that

$$
F_{n}=\left\{\left(i_{1}, \ldots, i_{d}\right) \mid-a_{n}<i_{j} \leq a_{n}, j=1, \ldots, d\right\},
$$


for each $n$. Suppose that we have defined the subsets $C_{1}, F_{1}, \ldots, C_{n-1}, F_{n-1}$. Our purpose is to construct $C_{n}$ and $F_{n}$. Choose $c_{n} \in \mathbb{Z}^{d}$ such that $\left(c_{n}+F_{n-1}\right) \cap F_{n-1}=\emptyset$, $\operatorname{dist}\left(c_{n}, \delta_{n}\right)<\epsilon_{n}$ and

$$
\begin{gathered}
\max _{f \in F_{n-1}} \operatorname{dist}\left(c_{n}, c_{n}+f\right)<\epsilon_{n}, \\
\pi\left(c_{n}\right) \notin L_{n}^{+} .
\end{gathered}
$$

For that use the fact that $\delta_{n} \notin \overline{L_{n}^{+}}$. We now let $C_{n}:=\left\{0, c_{n}\right\}$ and define $F_{n}$ to be a huge symmetric cube in $\mathbb{Z}^{d}$ that contains $F_{n-1}+C_{n}$. Continuing this construction procedure an infinite number of times we obtain infinite sequences $\left(F_{n}\right)_{n=0}^{\infty}$ and $\left(C_{n}\right)_{n=1}^{\infty}$. It is easy to see that (I)-(IV) and (4.1) are all satisfied. Let $T=\left(T_{\gamma}\right)_{\gamma \in \mathbb{Z}^{d}}$ denote the associated $(C, F)$-action. It is free and of rank one. Let $(X, \mu)$ be the space of this action.

We first show that $D \subset \mathcal{R}(T)$. Take $\delta \in D, \epsilon>0$ and a cylinder $B \subset X$. Then there are an infinite number of $n>0$ such that $\delta=\delta_{n}$ and hence $\operatorname{dist}\left(c_{n}, \delta\right)<\epsilon_{n}<\epsilon$. If $n$ is large enough, $B=\left[B_{n-1}\right]_{n-1}$ for some subset $B_{n-1} \subset F_{n-1}$. Since $\left[B_{n-1}\right]_{n} \subset\left[B_{n-1}\right]_{n-1}$ and $T_{c_{n}}\left[B_{n-1}\right]_{n}=\left[c_{n}+B_{n-1}\right]_{n} \subset\left[B_{n-1}\right]_{n-1}$ with $\mu\left(\left[B_{n-1}\right]_{n}\right)=0.5 \mu\left(\left[B_{n-1}\right]_{n-1}\right)$,

$$
\mu\left(T_{c_{n}} B \cap B\right) \geq \mu\left(T_{c_{n}}\left[B_{n-1}\right]_{n} \cap[B]_{n-1}\right)=\mu\left(\left[B_{n-1}+c_{n}\right]_{n}\right)=0.5 \mu(B) .
$$

Since each subset of finite measure in $X$ can be approximated with a cylinder up to an arbitrary positive real, we deduce that $\delta \in \mathcal{R}(T)$.

We now show that $\mathcal{R}(T) \subset \Delta$. It follows from (5.1) that $\left\{c_{n}\right\} \gg F_{n-1}$ as $n \rightarrow \infty$. Hence, by Proposition 5.1(iii), $\mathcal{R}(T) \subset \bigcap_{n=1}^{\infty} \overline{\left\{\pi\left(c_{m}\right) \mid m \geq n\right\}}$. Applying (5.2), we obtain that $\pi\left(c_{m}\right) \notin L_{m}^{+} \supset L_{n}^{+} \supset L_{n}$, for each $m \geq n$. Hence $\mathcal{R}(T) \cap L_{n}=\emptyset$ for each $n$, which yields $\mathcal{R}(T) \subset \Delta$.

It remains to consider the case where $\Delta=\emptyset$. Fix $\theta \in P\left(\mathbb{R}^{d}\right)$. Suppose that we have defined the subsets $C_{1}, F_{1}, \ldots, C_{n-1}, F_{n-1}$. Choose $c_{n} \in \mathbb{Z}^{d}$ such that $\left(c_{n}+F_{n-1}\right) \cap$ $F_{n-1}=\emptyset,(5.1)$ is satisfied,

$$
\begin{aligned}
& \pi\left(c_{n}\right) \text { is up to } \epsilon_{n} \text { close to } \theta \text { (in the metric on } P\left(\mathbb{R}^{d}\right) \text { ) and } \\
& \qquad \min _{f \in F_{n-1}-F_{n-1}} \operatorname{dist}\left(c_{n}+f, \theta\right)>10 .
\end{aligned}
$$

We now let $C_{n}:=\left\{0, c_{n}\right\}$ and define $F_{n}$ to be a huge symmetric cube in $\mathbb{Z}^{d}$ that contains $F_{n-1}+C_{n}$. Continuing an infinite number of times, we obtain infinite sequences $\left(F_{n}\right)_{n=0}^{\infty}$ and $\left(C_{n}\right)_{n=1}^{\infty}$. It is easy to see that (I)-(IV) and (4.1) are all satisfied. Let $T=\left(T_{\gamma}\right)_{\gamma \in \mathbb{Z}^{d}}$ denote the associated $(C, F)$-action. It follows from Proposition 5.1(iii), (5.1) and (5.3) that $\mathcal{R}(T) \subset\{\theta\}$. If $T$ were recurrent along $\theta$, then there is $\gamma \in \mathbb{Z}^{d}$ such that $\gamma \neq 0$, $\operatorname{dist}(\gamma, \theta)<0.1$ and $\mu\left([0]_{n} \cap T_{\gamma}[0]_{n}\right)>0$. It follows, from Lemma 4.1, that there is $l>n$ such that $\gamma \in F_{l-1}-F_{l-1}+c_{l}$. This contradicts (5.4). Thus we obtain that $\mathcal{R}(T)=\emptyset$.

THEOREM 5.3. There is a rank-one free infinite measure-preserving action $T$ of $\mathbb{Z}^{d}$ such that $\mathcal{R}(T)=P\left(\mathbb{R}^{d}\right)$.

Proof. Given $t \in \mathbb{N}$ and $N>0$, we let

$$
\mathcal{K}_{t, N}:=\left\{\left(i_{1}, \ldots, i_{d}\right) \in \mathbb{Z}^{d}|| i_{j} \mid<N \text { and } t \text { divides } i_{j}, j=1, \ldots, d\right\} .
$$


Then, for each $\epsilon>0$ and each integer $t>0$, there is $N>0$ such that

$$
\sup _{\delta \in P\left(\mathbb{R}^{m}\right)} \min _{0 \neq \gamma \in \mathcal{K}_{t, N}} \operatorname{dist}(\gamma, \delta)<\epsilon .
$$

Fix a sequence of positive reals $\epsilon_{n}, n \in \mathbb{N}$, decreasing to zero. We will construct, inductively, the sequences $\left(F_{n-1}\right)_{n>0}$ and $\left(C_{n}\right)_{n>0}$ that satisfy (I)-(IV) and (4.1). As usual, $F_{0}=\{0\}$. Suppose we have defined $\left(F_{j}, C_{j}\right)_{j=1}^{n}$. Suppose that $F_{n}$ is a symmetric cube. Denote by $t_{n}$ the length of an edge of this cube. We now construct $C_{n+1}$ and $F_{n+1}$.

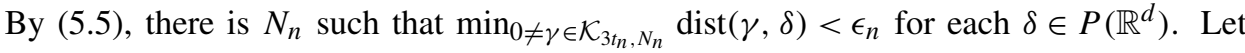
$C_{n+1}:=\mathcal{K}_{3 t_{n}, M_{n}}$, where $M_{n}$ is an integer large enough so that

$$
\#\left\{\gamma \in \mathcal{K}_{3 t_{n}, M_{n}} \mid \gamma+\mathcal{K}_{3 t_{n}, N_{n}} \subset \mathcal{K}_{3 t_{n}, M_{n}}\right\}>0.5 \# \mathcal{K}_{3 t_{n}, M_{n}} .
$$

Now let $F_{n+1}$ be a huge symmetric cube in $\mathbb{Z}^{d}$ such that $F_{n+1} \supset F_{n}+C_{n+1}$. Continuing this construction process an infinite number of times, we define the infinite sequences $\left(F_{n}\right)_{n \geq 0}$ and $\left(C_{n}\right)_{n \geq 1}$, as desired. Let $T$ be the $(C, F)$-action of $\mathbb{Z}^{d}$ associated with these sequences. It is free and of rank one. Denote by $(X, \mu)$ the space of this action. We claim that $\mathcal{R}(T)=P\left(\mathbb{R}^{d}\right)$. Indeed, take $\epsilon>0, \delta \in P\left(\mathbb{R}^{d}\right)$ and a cylinder $B \subset X$. Then there is $n>0$ and a subset $B_{n} \subset F_{n}$ such that $B=\left[B_{n}\right]_{n}$ and $\epsilon_{n}<\epsilon$. There is $\gamma \in \mathcal{K}_{3 t_{n}, N_{n} \backslash\{0\}}$ such that $\operatorname{dist}(\gamma, \delta)<\epsilon_{n}$. By (5.6), \#(C $\left.C_{n+1} \cap\left(C_{n+1}-\gamma\right)\right) \geq 0.5 \# C_{n+1}$. Therefore

$$
\begin{aligned}
\mu\left(T_{\gamma} B \cap B\right) & \geq \mu\left(T_{\gamma}\left[B_{n}+\left(C_{n+1} \cap\left(C_{n+1}-\gamma\right)\right)\right]_{n+1} \cap\left[B_{n}\right]_{n}\right) \\
& =\mu\left(\left[B_{n}+\left(C_{n+1} \cap\left(C_{n+1}+\gamma\right)\right)\right]_{n+1}\right) \\
& \geq 0.5 \mu(B) .
\end{aligned}
$$

The standard approximation argument implies that $T$ is recurrent along $\delta$.

Remark 5.4.

(i) If we choose $M_{m}$ in the above construction large enough so that the inequality

$$
\#\left\{\gamma \in \mathcal{K}_{3 t_{n}, M_{n}} \mid \gamma+\mathcal{K}_{3 t_{n}, N_{n}} \subset \mathcal{K}_{3 t_{n}, M_{n}}\right\}>\left(1-n^{-1}\right) \# \mathcal{K}_{3 t_{n}, M_{n}}
$$

holds in place of (5.6), then the corresponding $(C, F)$-action $T$ will possess the stronger property $\mathcal{R} i(T)=P\left(\mathbb{R}^{d}\right)$.

(ii) In a similar way, the statement of Theorem 5.2 remains true if we replace $\mathcal{R}(T)$ with $\mathcal{R i}(T)$.

\section{The generic $\mathbb{Z}^{d}$-action is recurrent in every direction}

Let $(X, \mu)$ be a $\sigma$-finite non-atomic standard measure space. We recall that the group of all $\mu$-preserving invertible transformations of $X$ is denoted by $\operatorname{Aut}(X, \mu)$. It is endowed with the weak topology under which it is a Polish space. For a nilpotent Lie group $G$, we denote by $\mathcal{A}_{\mu}^{G}$ the set of all $\mu$-preserving actions of $G$ on $(X, \mu)$. We consider every element $A \in$ $\mathcal{A}_{\mu}^{G}$ as a continuous homomorphism $g \mapsto A_{g}$ from $G$ to $\operatorname{Aut}(X, \mu)$. The $\operatorname{group} \operatorname{Aut}(X, \mu)$ acts on $\mathcal{A}_{\mu}^{G}$ by conjugation: i.e. $(S \cdot A)_{g}:=S A_{g} S^{-1}$ for all $g \in G, S \in \operatorname{Aut}(X, \mu)$ and $A \in \mathcal{A}_{\mu}^{G}$. We endow $\mathcal{A}_{\mu}^{G}$ with the compact-open topology: i.e. the topology of uniform convergence on the compact subsets of $G$.

The following lemma is well known. We state it without proof. 
Lemma 6.1. $\mathcal{A}_{\mu}^{G}$ is a Polish space. The action of $\operatorname{Aut}(X, \mu)$ on this space is continuous.

Let $S^{1}$ be the unit sphere in $\mathfrak{g}$ and let $K:=\exp \left(S^{1}\right)$.

Lemma 6.2. Let $\mu(X)=1$. Then the subset

$$
\mathcal{Z}:=\left\{A \in \mathcal{A}_{\mu}^{G} \mid h\left(A_{g}\right)=0 \text { for each } g \in K\right\}
$$

is an invariant $G_{\delta}$ in $\mathcal{A}_{\mu}^{G}$.

Proof. Denote by $\mathcal{P}$ the set of all finite partitions of $X$. Fix a countable subset $\mathcal{P}_{0} \subset \mathcal{P}$ which is dense in $\mathcal{P}$ in the natural topology. For each $P \in \mathcal{P}_{0}$ and $n>0$, the map

$$
\mathcal{A}_{\mu}^{G} \times K \ni(A, g) \mapsto H\left(P \mid \bigvee_{j=1}^{n} A_{g}^{-j} P\right) \in \mathbb{R}
$$

is continuous. Therefore the map

$$
m_{P, n}: \mathcal{A}_{\mu}^{G} \ni A \mapsto m_{P, n}(A):=\max _{g \in K} H\left(P \mid \bigvee_{j=1}^{n} A_{g}^{-j} P\right) \in \mathbb{R}
$$

is well defined and continuous. Hence the subset

$$
\mathcal{Z}^{\prime}:=\bigcap_{P \in \mathcal{P}_{0}} \bigcap_{r=1}^{\infty} \bigcap_{N=1}^{\infty} \bigcup_{l>N}\left\{A \in \mathcal{A}_{\mu}^{G} \mid m_{P, l}(A)<1 / r\right\}
$$

is a $G_{\delta}$ in $\mathcal{A}_{\mu}^{G}$. We now show that $\mathcal{Z}^{\prime}=\mathcal{Z}$. It is easy to see that $\mathcal{Z}^{\prime} \subset \mathcal{Z}$ because $h\left(A_{g}\right)=\sup _{P \in \mathcal{P}_{0}} H\left(P \mid \bigvee_{j=1}^{\infty} A_{g}^{-j} P\right)$. Conversely, let $A \in \mathcal{Z}$. Fix $P \in \mathcal{P}_{0}, r>1$ and $N>0$. Then for each $g \in K$, there is $l_{g}>N$ such that $H\left(P \mid \bigvee_{j=1}^{l_{g}} A_{g}^{-j} P\right)<1 / r$. Of course, this inequality holds in a neighborhood of $g$ in $G$. Since $K$ is compact and the map $\mathbb{N} \ni n \mapsto H\left(P \mid \bigvee_{j=1}^{n} A_{g}^{-j} P\right)$ decreases, there is $l>N$ such that $H\left(P \mid \bigvee_{j=1}^{l} A_{g}^{-j} P\right)<$ $1 / r$ for all $g \in K$ : i.e. $m_{P, l}(A)<1 / r$. This means that $A \in \mathcal{Z}^{\prime}$. It is obvious that $\mathcal{Z}$ is $\operatorname{Aut}(X, \mu)$-invariant.

Let $\Gamma$ be a co-compact lattice in $G$. Fix a a cross section $s: G / \Gamma \rightarrow G$ of the natural projection $G \rightarrow G / \Gamma$ such that the subset $s(G / \Gamma)$ is relatively compact in $G$. Denote by $h_{s}$ the corresponding one-cocycle. Given a $\Gamma$-action $T$ on $(X, \mu)$, we construct (via $\left.h_{s}\right)$ the induced $G$-action $\widetilde{T}$ on the space $(G / \Gamma \times X, \lambda \times \mu)$. In the following lemma we show that the 'inducing' functor is continuous.

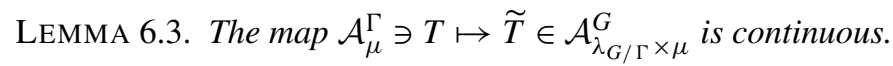

Idea of the proof. It is enough to note that, for each compact subset $K \subset G$, the set $F:=$ $\left\{h_{s}(g, y) \mid g \in K, y \in G / \Gamma\right\} \subset \Gamma$ is finite. Therefore, given two $\Gamma$-actions $T$ and $T^{\prime}$, if the transformation $T_{\gamma}$ is 'close' to $T_{\gamma}^{\prime}$ for each $\gamma \in F$, then the transformation $\widetilde{T}_{g}$ is 'close' to $\widetilde{T}_{g}^{\prime}$ uniformly on $K$.

From now on let $\mu(X)=\infty$. Denote by $\left(X^{\bullet}, \mu^{\bullet}\right)$ the Poisson suspension of $(X, \mu)$. Given $R \in \operatorname{Aut}(X, \mu)$, let $R^{\bullet}$ stand for the Poisson suspension of $R$ (see [Ro, Ja-Ru]). We note that $\operatorname{Aut}\left(X^{\bullet}, \mu^{\bullet}\right)$ is a topological $\operatorname{Aut}(X, \mu)$-module. 
Lemma 6.4. The map $\operatorname{Aut}(X, \mu) \ni R \mapsto R^{\bullet} \in \operatorname{Aut}\left(X^{\bullet}, \mu^{\bullet}\right)$ is a continuous homomorphism.

Idea of the proof. Let $U_{R}$ and $U_{R}$ • denote the Koopman unitary operators generated by $R$ and $R^{\bullet}$, respectively. Then it is enough to note that $U_{R^{\bullet}}$ is unitarily equivalent in a canonical way to the exponent $\bigoplus_{n \geq 0} U_{R}^{\odot n}$ (see [Ne, Ro]) and the map $U_{R} \mapsto U_{R}^{\odot n}$ is continuous in the weak operator topology for each $n$.

LEMMA 6.5. Let a transformation $R \in \operatorname{Aut}(X, \mu)$ be non-conservative. If there is an ergodic countable transformation subgroup $N \subset \operatorname{Aut}(X, \mu)$ such that

$$
\left\{S R^{n} x \mid n \in \mathbb{Z}\right\}=\left\{R^{n} S x \mid n \in \mathbb{Z}\right\} \quad \text { at almost every } x \in X \text { for each } S \in N,
$$

then $R^{\bullet}$ is a Bernoulli transformation of infinite entropy.

Proof. We consider Hopf decomposition of $X$ : i.e. a partition of $X$ into two $R$-invariant subsets $X_{d}$ and $X_{c}$ such that the restriction of $R$ to $X_{d}$ is totally dissipative and the restriction of $R$ to $X_{d}$ is conservative (see [Aa]). By the hypothesis, $\mu\left(X_{d}\right)>0$. It follows, from (6.1), that $X_{d}$ is invariant under $N$. Since $N$ is ergodic, $\mu\left(X_{c}\right)=0$ : i.e. $R$ is totally dissipative so there is a subset $W \subset X$ such that $X=\bigcup_{n \in \mathbb{Z}} R^{n} W(\bmod 0)$ and $R^{n} W \cap T^{m} W=\emptyset$ if $n \neq m$. Therefore $R^{\bullet}$ is Bernoulli [Ro]. Since $\mu \uparrow W$ is not purely atomic, $h\left(R^{\bullet}\right)=\infty[\mathbf{R o}]$.

We now state the main result of this section.

THEOREM 6.6. The subset $\mathcal{V}$ of $\mathbb{Z}^{d}$-actions $T$ on $(X, \mu)$ with $\mathcal{R}(T)=P\left(\mathbb{R}^{d}\right)$ is residual in $\mathcal{A}_{\mu}^{\mathbb{Z}^{d}}$

Proof. Let $\lambda$ denote Haar measure on the torus $\mathbb{R}^{d} / \mathbb{Z}^{d}$. It follows, from Lemmata 5.3 and 5.4 , that the mapping

$$
\mathcal{A}_{\mu}^{\mathbb{Z}^{d}} \ni T \mapsto \widetilde{T}^{\bullet} \in \mathcal{A}_{\lambda \times \mu}^{\mathbb{R}^{d}}
$$

is continuous. Let $\mathcal{Z}:=\left\{A \in \mathcal{A}_{(\lambda \times \mu)}^{\mathbb{R}^{d}} \mid h\left(A_{g}\right)=0\right.$ for each $\left.g \in \mathbb{R}^{d}\right\}$. By Lemma 6.2, $\mathcal{Z}$ is a $G_{\delta}$ in $\mathcal{A}_{\mu}^{\mathbb{R}^{d}}$. Hence the subset $\mathcal{W}:=\left\{T \in \mathcal{A}_{\mu}^{\mathbb{Z}^{d}} \mid \widetilde{T}^{\bullet} \in \mathcal{Z}\right\}$ is a $G_{\delta}$ in $\mathcal{A}_{\mu}^{\mathbb{Z}^{d}}$. Of course, $\mathcal{W}$ is $\operatorname{Aut}(X, \mu)$-invariant. It is well known that the subset $\mathcal{E}:=\left\{T \in \mathcal{A}_{\mu}^{\mathbb{Z}^{d}} \mid T\right.$ is ergodic $\}$ is an $\operatorname{Aut}(X, \mu)$-invariant $G_{\delta}$ in $\mathcal{A}_{\mu}^{\mathbb{Z}^{d}}$. Hence the intersection $\mathcal{W} \cap \mathcal{E}$ is also an $\operatorname{Aut}(X, \mu)$ invariant $G_{\delta}$ in $\mathcal{A}_{\mu}^{\mathbb{Z}^{d}}$. Take an action $T \in \mathcal{A}^{\mathbb{Z}^{d}} \cap \mathcal{E}$ and a line $\theta \in P\left(\mathbb{R}^{d}\right)$. If $\theta \notin \mathcal{R}(T)$, then $\theta \notin \mathcal{R}(\widetilde{T})$. Since $T$ is ergodic, $\widetilde{T}$ is also ergodic. Hence the $\mathbb{Q}^{d}$-action $\left(\widetilde{T}_{q}\right)_{q \in \mathbb{Q}^{d}}$ is also ergodic. Then, by Lemma $6.5, h\left(\widetilde{T}_{r}^{\bullet}\right)=\infty$ for each $r \in \theta, r \neq 0$. Therefore $T \notin \mathcal{W}$. This yields that $\mathcal{W} \cap \mathcal{E} \subset \mathcal{V}$. It remains to show that $\mathcal{W} \cap \mathcal{E}$ is dense in $\mathcal{A}_{\mu}^{\mathbb{Z}^{d}}$. Let $T$ be an ergodic free action of $\mathbb{Z}^{d}$ such that $\mathcal{R} i(T)=P\left(\mathbb{R}^{d}\right)$ (see Remark 5.4(i) and Theorem 5.3). By Theorem 3.1, $\mathcal{R} i(\widetilde{T})=P\left(\mathbb{R}^{d}\right)$. Then, in view of Lemma 6.4 , for each $g \in \mathbb{R}^{d}$, the transformation $\widetilde{T}_{g}^{\bullet}$ is rigid. Hence $h\left(\widetilde{T}_{g}^{\bullet}\right)=0$. Thus $T \in \mathcal{W} \cap \mathcal{E}$. It follows, from the Rokhlin lemma for the infinite measure-preserving free $\mathbb{Z}^{d}$-actions, that the conjugacy class of $T$ (i.e. the $\operatorname{Aut}(X, \mu)$-orbit of $T$ ) is dense in $\mathcal{A}_{\mu}^{\mathbb{Z}^{d}}$ (see, e.g., [DaSi]). Of course, the conjugacy class of $T$ is a subset of $\mathcal{W} \cap \mathcal{E}$. 
Using some ideas from the proof of the above theorem, we can prove the following proposition.

Proposition 6.7. There is a Poisson action $\dagger V$ of $\mathbb{R}^{d}$ of zero entropy such that, for each $0 \neq g \in \mathbb{R}^{m}$, the transformation $V_{g}$ is Bernoullian and of infinite entropy.

Proof. By Theorem 5.2, there exists rank-one (by cubes) infinite measure-preserving actions $T$ of $\mathbb{Z}^{d}$ such that $\mathcal{R}(T)=\emptyset$. Then $\widetilde{T}^{\bullet}$ is a Poisson (finite measure-preserving) action of $\mathbb{R}^{d}$. We note that $h\left(\widetilde{T}^{\bullet}\right)=h\left(\widetilde{T} \bullet \mathbb{Z}^{d}\right)=h\left(\left(\widetilde{T}\left\lceil\mathbb{Z}^{d}\right)^{\bullet}\right)\right.$. We note $\widetilde{T}\left\lceil\mathbb{Z}^{d}=I \times T\right.$, where $I$ denotes the trivial action of $\mathbb{Z}^{d}$ on the torus $\left(\mathbb{R}^{d} / \mathbb{Z}^{d}, \lambda\right)$. It follows, from [Ja-Ru], that $h\left((I \times T)^{\bullet}\right)=h\left(T^{\bullet}\right)$. Since $T$ is of rank one, $h\left(T^{\bullet}\right)=0$, by [Ja-Ru] $\ddagger$. Thus we obtain that $h\left(\widetilde{T}^{\bullet}\right)=0$. On the other hand, arguing as in the proof of Theorem 6.6, we deduce, from Theorem 3.1 and Lemma 6.5, that, for each $g \in \mathbb{R}^{d} \backslash\{0\}$, the transformation $\widetilde{T}_{g}^{\bullet}$ is Bernoulli and of infinite entropy.

In a similar way, using Remark 5.4(ii), we can show the following more general statement.

Proposition 6.8. Let $\Delta$ be a $G_{\delta}$-subset of $P\left(\mathbb{R}^{d}\right)$ and let $D$ be a countable subset of $\Delta$. Then there is a Poisson action $V$ of $\mathbb{R}^{d}$ of zero entropy such that, for each nonzero $g \notin \bigcup_{\theta \in \Delta} \theta$, the transformation $V_{g}$ is Bernoulli and of infinite entropy and, for each $g \in \bigcup_{\theta \in D} \theta$, the transformation $V_{g}$ is rigid (and hence of zero entropy).

\section{Directional recurrence for actions of the Heisenberg group}

Consider now the three-dimensional real Heisenberg group $H_{3}(\mathbb{R})$, which is perhaps the simplest example of a non-commutative simply connected nilpotent Lie group. We recall that

$$
H_{3}(\mathbb{R})=\left\{\left(\begin{array}{ccc}
1 & t_{1} & t_{3} \\
0 & 1 & t_{2} \\
0 & 0 & 1
\end{array}\right) \mid t_{1}, t_{2}, t_{3} \in \mathbb{R}\right\}
$$

We introduce the notation

$$
a(t):=\left(\begin{array}{lll}
1 & t & 0 \\
0 & 1 & 0 \\
0 & 0 & 1
\end{array}\right), \quad b(t):=\left(\begin{array}{ccc}
1 & 0 & 0 \\
0 & 1 & t \\
0 & 0 & 1
\end{array}\right), \quad c(t):=\left(\begin{array}{lll}
1 & 0 & t \\
0 & 1 & 0 \\
0 & 0 & 1
\end{array}\right) .
$$

Then the maps $\mathbb{R} \ni t \mapsto a(t) \in H_{3}(\mathbb{R}), \mathbb{R} \ni t \mapsto b(t) \in H_{3}(\mathbb{R}), \mathbb{R} \ni t \mapsto c(t) \in H_{3}(\mathbb{R})$ are continuous homomorphisms, the subset $\{c(t) \mid t \in \mathbb{R}\}$ is the center of $H_{3}(\mathbb{R}), a\left(t_{1}\right) b\left(t_{2}\right)=$ $b\left(t_{2}\right) a\left(t_{1}\right) c\left(t_{1} t_{2}\right)$ for all $t_{1}, t_{2} \in \mathbb{R}$ and

$$
\left(\begin{array}{ccc}
1 & t_{1} & t_{3} \\
0 & 1 & t_{2} \\
0 & 0 & 1
\end{array}\right)=c\left(t_{3}\right) b\left(t_{2}\right) a\left(t_{1}\right) \quad \text { for all } t_{1}, t_{2}, t_{3} \in \mathbb{R}
$$

\footnotetext{
$\dagger$ We recall that a probability-preserving action of a group $G$ is called Poisson if it is isomorphic to the Poisson suspension of an infinite measure-preserving action of $G$.

$\ddagger$ This fact was proved in [Ja-Ru] only for $d=1$. However, in the general case, the proof is similar.
} 
We also note that the Lie algebra of $H_{3}(\mathbb{R})$ is

$$
\mathfrak{h}_{3}(\mathbb{R}):=\left\{\left(\begin{array}{ccc}
0 & t_{1} & t_{3} \\
0 & 0 & t_{2} \\
0 & 0 & 0
\end{array}\right) \mid \alpha, \beta, \gamma \in \mathbb{R}\right\} .
$$

The exponential map exp $: \mathfrak{h}_{3}(\mathbb{R}) \rightarrow H_{3}(\mathbb{R})$ is given by the formula

$$
\exp \left(\begin{array}{ccc}
0 & t_{1} & t_{3} \\
0 & 0 & t_{2} \\
0 & 0 & 0
\end{array}\right)=\left(\begin{array}{ccc}
1 & t_{1} & t_{3}+\frac{t_{1} t_{2}}{2} \\
0 & 1 & t_{2} \\
0 & 0 & 1
\end{array}\right) .
$$

The adjoint action of $H_{3}(\mathbb{R})$ on $\mathfrak{h}_{3}(\mathbb{R})$ is given by the formula

$$
\left(\begin{array}{lll}
1 & x & z \\
0 & 1 & y \\
0 & 0 & 1
\end{array}\right) \cdot\left(\begin{array}{ccc}
0 & \alpha & \gamma \\
0 & 0 & \beta \\
0 & 0 & 0
\end{array}\right)=\left(\begin{array}{ccc}
0 & \alpha & \gamma+x \beta-y \alpha \\
0 & 0 & \beta \\
0 & 0 & 0
\end{array}\right)
$$

We also give an example of a right-invariant metric $d$ on $H_{3}(\mathbb{R})$ : that is,

$$
d\left(c\left(t_{3}\right) b\left(t_{2}\right) a\left(t_{1}\right), c\left(t_{3}^{\prime}\right) b\left(t_{2}^{\prime}\right) a\left(t_{1}^{\prime}\right)\right):=\left|t_{1}-t_{1}^{\prime}\right|+\left|t_{2}-t_{2}^{\prime}\right|+\left|t_{3}-t_{3}^{\prime}+t_{2}^{\prime}\left(t_{1}^{\prime}-t_{1}\right)\right| .
$$

Let $\Gamma$ be a lattice in $H_{3}(\mathbb{R})$. It is well known (see, e.g., [DaLe]) that there is $k>0$ such that $\Gamma$ is automorphic to the lattice

$$
\left\{c\left(n_{3} / k\right) b\left(n_{2}\right) a\left(n_{1}\right) \mid n_{1}, n_{2}, n_{3} \in \mathbb{Z}\right\} .
$$

From now on, we will assume that $k=1$ and hence

$$
\Gamma=H_{3}(\mathbb{Z}):=\left\{c\left(n_{3}\right) b\left(n_{2}\right) a\left(n_{1}\right) \mid n_{1}, n_{2}, n_{3} \in \mathbb{Z}\right\} .
$$

Let $F_{n}:=\left\{c\left(j_{3}\right) b\left(j_{2}\right) a\left(j_{1}\right)|| j_{1}\left|<L_{n},\right| j_{2}\left|<L_{n},\right| j_{3} \mid<M_{n}\right\}$, where $L_{n}$ and $M_{n}$ are positive integers. It is easy to verify that if $L_{n} \rightarrow \infty, M_{n} \rightarrow \infty$ and $L_{n} / M_{n} \rightarrow 0$ as $n \rightarrow \infty$, then $\left(F_{n}\right)_{\geq 1}$ is a Følner sequence in $H_{3}(\mathbb{Z})$.

In the following three theorems, we construct rank-one actions of $H_{3}(\mathbb{Z})$ with various sets of recurrence and rigidity: empty, countable and uncountable.

THEOREM 7.1. There is a rank-one free infinite measure-preserving action $\mathrm{T}$ of $\mathrm{H}_{3}(\mathbb{Z})$ such that $\mathcal{R}(T)=\emptyset$.

Proof. Let $C_{n}:=\left\{1, a\left(t_{n}\right)\right\}$, where $\left(t_{n}\right)_{n \in \mathbb{N}}$ is a sequence of integers that grows fast, and let $\left(F_{n}\right)_{n \geq 0}$ be a Følner sequence in $H_{3}(\mathbb{R})$ such that (I)-(IV) and (4.1) are satisfied and, in addition, $C_{n} \backslash\{1\} \gg C_{1} \cdots C_{n-1}$ as $n \rightarrow \infty$. Denote by $T$ the $(C, F)$-action of $H_{3}(\mathbb{Z})$ associated with $\left(C_{n}, F_{n-1}\right)_{n \in \mathbb{N}}$. Let $\theta \in P\left(\mathfrak{h}_{3}(\mathbb{R})\right)$ stand for the line in $\mathfrak{h}_{3}(\mathbb{R})$ that passes through the vector $\left(\begin{array}{lll}0 & 1 & 0 \\ 0 & 0 & 0 \\ 0 & 0 & 0\end{array}\right)$. Since $\pi\left(\log a\left(t_{n}\right)\right)=\theta$, we deduce, from Proposition 4.2(iii), that

$$
\mathcal{R}(T) \subset \bigcap_{\gamma \in \Gamma} \gamma \cdot\left(\bigcap_{n=1}^{\infty} \overline{\left\{\pi\left(\log a\left(t_{m}\right)\right) \mid m \geq n\right\}}\right) \subset \bigcap_{\gamma \in \Gamma}\{\gamma \cdot \theta\}=\emptyset .
$$


Given $t \in \mathbb{R}$, let $\theta_{t} \in P\left(\mathfrak{h}_{3}(\mathbb{R})\right)$ be the line in $\mathfrak{h}_{3}(\mathbb{R})$ that passes through the vector $\left(\begin{array}{lll}0 & 1 & t \\ 0 & 0 & 0 \\ 0 & 0 & 0\end{array}\right)$. Then $\exp \left(\theta_{t}\right) \ni c(t) a(1)$. We also denote by $\theta_{\infty}$ the line in $\mathfrak{h}_{3}(\mathbb{R})$ that passes through the vector $\left(\begin{array}{lll}0 & 0 & 1 \\ 0 & 0 & 0 \\ 0 & 0 & 0\end{array}\right)$. Of course, the set $\left\{\theta_{l} \mid l \in \mathbb{Z}\right\}$ is the $H_{3}(\mathbb{Z})$-orbit $\left\{\gamma \cdot \theta_{0} \mid \gamma \in\right.$ $H_{3}(\mathbb{Z}\}$ of $\theta_{0}$. The point $\theta_{\infty}$ is the only limit point of this orbit in $\left.P(\mathfrak{h})_{3}(\mathbb{R})\right)$. In a similar way, the set $\left\{\theta_{t} \mid t \in \mathbb{R}\right\}$ is the $H_{3}(\mathbb{R})$-orbit of $\theta_{0}$. The closure of this orbit is the union of this orbit with the limit point $\theta_{\infty}$.

THEOREM 7.2. There is a rank-one free infinite measure-preserving action $\mathrm{T}$ of $\mathrm{H}_{3}(\mathbb{Z})$ such that $\mathcal{R}(T)=\left\{\theta_{l} \mid l \in \mathbb{Z}\right\} \cup\left\{\theta_{\infty}\right\}$. Therefore $\mathcal{E} \mathcal{R}(T)=\left\{\theta_{\infty}\right\}$ and hence $\mathcal{R}(T) \neq$ $\mathcal{E R}(T)$.

Proof. We let

$$
\begin{aligned}
& F_{n}:=\left\{c\left(j_{3}\right) b\left(j_{2}\right) a\left(j_{1}\right)|| j_{1}\left|<L_{n},\right| j_{2}\left|<L_{n},\right| j_{3} \mid<M_{n}\right\} \quad \text { and } \\
& C_{n}:=\left\{c\left(i k_{n}\right) a\left(j k_{n}\right) \mid j=0,1 \text { and }|i| \leq I_{n}\right\},
\end{aligned}
$$

where $\left(L_{n}\right)_{n \geq 1},\left(M_{n}\right)_{n \geq 1},\left(k_{n}\right)_{n \geq 1}$ and $\left(I_{n}\right)_{n \geq 1}$ are sequences of integers chosen in such a way such that:

(•) (I)-(IV) from $\$ 4$ and (4.1) are satisfied;

(*) $C_{n} \backslash\{1\} \gg C_{1} \cdots C_{n-1}$ as $n \rightarrow \infty$;

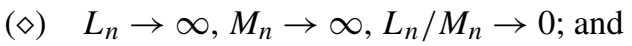

(০) $I_{n} \rightarrow+\infty, L_{n-1} / I_{n} \rightarrow 0$.

Denote by $T$ the $(C, F)$-action of $H_{3}(\mathbb{Z})$ associated with $\left(C_{n}, F_{n-1}\right)_{n \in \mathbb{N}}$. It is well defined in view of $(\bullet)$. Moreover, $\left(F_{n}\right)_{n \geq 1}$ is a Følner sequence in $H_{3}(\mathbb{Z})$ in view of $(\diamond)$. It is standard to verify that

$$
\overline{\bigcup_{m>n} \pi\left(\log \left(C_{m} C_{m}^{-1} \backslash\{1\}\right)\right)}=\left\{\theta_{l} \mid l \in \mathbb{Z}\right\} \cup\left\{\theta_{\infty}\right\}
$$

for each $n>0$. Hence, by Proposition 4.2(ii), $\mathcal{R}(T) \subset\left\{\theta_{n} \mid n \in \mathbb{Z}\right\} \cup\left\{\theta_{\infty}\right\}$. In view of Remark 2.4(ii), to prove the converse inclusion it suffices to show that $\theta_{1}, \theta_{\infty} \in \mathcal{R}(T)$. For $n \geq 1$, take a subset $D \subset F_{n-1}$. It follows, from the definition of $F_{n-1}$, that, for each $\gamma \in D$, there is $j \in \mathbb{Z}$ such that $|j|<L_{n-1}$ and $a\left(k_{n}\right) \gamma a\left(-k_{n}\right)=\gamma c\left(j k_{n}\right)$. Let

$$
C_{n}^{\prime}:=\left\{w \in C_{n} \mid c\left(j k_{n}\right) a\left(k_{n}\right) w \in C_{n} \text { whenever }|j|<L_{n-1}\right\} .
$$

Then $C_{n}^{\prime}=\left\{c\left(i k_{n}\right)|| i\left|<I_{n},\right| i \pm L_{n-1} \mid<I_{n}\right\}$. Hence $\# C_{n}^{\prime} / \# C_{n} \rightarrow 1 / 2$ as $n \rightarrow \infty$, in view of (o), and hence

$$
\max _{D \subset F_{n-1}}\left|\mu\left([D]_{n-1}\right) / \mu\left(\left[D C_{n}^{\prime}\right]_{n}\right)-1 / 2\right| \rightarrow 0
$$

as $n \rightarrow \infty$. On the other hand, in view of (7.1), we have

$$
T_{a\left(k_{n}\right)}\left[D C_{n}^{\prime}\right]_{n}=\bigsqcup_{\gamma \in D} T_{a\left(k_{n}\right)}\left[\gamma C_{n}^{\prime}\right]_{n}=\bigsqcup_{\gamma \in D}\left[a\left(k_{n}\right) \gamma a\left(-k_{n}\right) a\left(k_{n}\right) C_{n}^{\prime}\right]_{n} \subset \bigsqcup_{\gamma \in D}\left[\gamma C_{n}\right]_{n} .
$$

Thus $T_{a\left(k_{n}\right)}\left[D C_{n}^{\prime}\right]_{n} \subset[D]_{n-1}$. Since $a\left(k_{n}\right) \in \exp \left(\theta_{1}\right)$ and (7.2) holds, it follows that $T$ is recurrent along $\theta_{1}$. To prove that $\theta_{\infty} \in \mathcal{R}(T)$, we let

$$
C_{n}^{\prime \prime}:=\left\{w \in C_{n} \mid c\left(k_{n}\right) w \in C_{n}\right\} .
$$

Then $\# C_{n}^{\prime \prime} / \# C_{n} \rightarrow 1$ and hence $\max _{D \subset F_{n-1}}\left|\mu\left([D]_{n-1}\right) / \mu\left(\left[D C_{n}^{\prime}\right]_{n}\right)-1\right| \rightarrow 0$ as $n \rightarrow \infty$. Moreover, $T_{c\left(k_{n}\right)}\left[D C_{n}^{\prime \prime}\right]_{n} \subset\left[D C_{n}\right]_{n}=[D]_{n-1}$. Hence $T$ is recurrent along $\theta_{\infty}$. 
THEOREM 7.3. There is a rank-one free infinite measure-preserving action $T$ of $H_{3}(\mathbb{Z})$ such that $\mathcal{R}(T)=\mathcal{R} i(T)=\left\{\theta_{t} \mid t \in \mathbb{R}\right\} \cup\left\{\theta_{\infty}\right\}=\mathcal{E} \mathcal{R}(T)=\mathcal{E} \mathcal{R} i(T)$.

Proof. Let

$$
\begin{aligned}
& F_{n}:=\left\{c\left(j_{3}\right) b\left(j_{2}\right) a\left(j_{1}\right)|| j_{1}\left|<L_{n},\right| j_{2}\left|<L_{n},\right| j_{3} \mid<M_{n}\right\}, \\
& C_{n}:=\left\{c\left(j k_{n}\right) a\left(i k_{n}\right)|| j\left|\leq l_{n} J_{n},\right| i \mid \leq l_{n} I_{n}\right\}, \\
& C_{n}^{0}:=\left\{c\left(j k_{n}\right) a\left(i k_{n}\right)|| j\left|\leq l_{n},\right| i \mid \leq l_{n}\right\},
\end{aligned}
$$

where $\left(L_{n}\right)_{n \geq 1},\left(M_{n}\right)_{n \geq 1},\left(k_{n}\right)_{n \geq 1},\left(I_{n}\right)_{n \geq 1},\left(J_{n}\right)_{n \geq 1}$ and $\left(l_{n}\right)_{n \geq 1}$ are sequences of integers such that $(\bullet),(*),(\diamond)$ hold,

$(\triangle) \sup _{t \in \mathbb{R} \cup\{\infty\}} \min _{1 \neq \gamma \in C_{n}^{0}} \operatorname{dist}\left(\gamma, \theta_{t}\right)<1 / n$ and

(ム) $\#\left(\left\{w \in C_{n} \mid \bigcup_{d \in F_{n-1}} \bigcup_{c \in C_{n}^{0}} d^{-1} c d w \subset C_{n}\right\}\right)>(1-1 / n) \# C_{n}$,

for each $n \in \mathbb{N}$. Denote by $T$ the $(C, F)$-action of $H_{3}(\mathbb{Z})$ associated with $\left(C_{n}, F_{n-1}\right)_{n \in \mathbb{N}}$. It is standard to verify that

$$
\overline{\bigcup_{m>n} \pi\left(\log \left(C_{m} C_{m}^{-1} \backslash\{1\}\right)\right)}=\left\{\theta_{t} \mid t \in \mathbb{R}\right\} \cup\left\{\theta_{\infty}\right\} .
$$

Hence, by Proposition 4.2(ii), $\mathcal{R}(T) \subset\left\{\theta_{t} \mid t \in \mathbb{R}\right\} \cup\left\{\theta_{\infty}\right\}$. To prove the converse inclusion, we take $\theta_{t}$ for some $t \in \mathbb{R} \cup\{\infty\}$. By $(\triangle)$, there is $\gamma \in C_{n}^{0} \backslash\{1\}$ such that $\operatorname{dist}\left(\gamma, \theta_{t}\right)<1 / n$. Let

$$
C_{n}^{\prime}:=\left\{w \in C_{n} \mid \bigcup_{d \in F_{n-1}} d^{-1} \gamma d w C_{n}^{0} \subset C_{n}\right\} .
$$

Then $\# C_{n}^{\prime} / \# C_{n}>1-1 / n$, in view of $(\mathbf{\Delta})$, and hence, for each subset $D \subset F_{n-1}$, we have $\mu\left([D]_{n-1} \backslash\left[D C_{n}^{\prime}\right]_{n}\right)<\mu\left([D]_{n}\right) / n$. On the other hand,

$$
T_{\gamma}\left[D C_{n}^{\prime}\right]_{n}=\bigsqcup_{d \in D} T_{\gamma}\left[d C_{n}^{\prime}\right]_{n}=\bigsqcup_{d \in D}\left[d d^{-1} \gamma d C_{n}^{\prime}\right]_{n} \subset \bigsqcup_{d \in D}\left[d C_{n}\right]_{n}=[D]_{n-1}
$$

It follows that $T$ is rigid along $\theta_{t}$. Thus we have shown that $\left\{\theta_{t} \mid t \in \mathbb{R}\right\} \cup\left\{\theta_{\infty}\right\} \subset \mathcal{R i}(T)$.

8. Some open problems and concluding remarks

(1) Which $G_{\delta}$-subsets of $P(\mathfrak{g})$ are realizable as $\mathcal{R}(T)$ or $\mathcal{R} i(T)$ for an ergodic infinite measure-preserving action $T$ of $\Gamma$ ? In particular, let $\theta \in P(\mathfrak{g})$. Is the subset $P(\mathfrak{g}) \backslash\{\theta\}$ is realizable? In the case where $G=\mathbb{R}^{2}$ and $\Gamma=\mathbb{Z}^{2}, P(\mathfrak{g})$ is homeomorphic to the circle. Is a proper arc of this circle realizable?

(2) Suppose that a subset of $P(\mathfrak{g})$ is realizable as $\mathcal{R}(T)$ or $\mathcal{R} i(T)$. Can we choose $T$ in the class of rank-one actions?

(3) In view of Theorem 3.1 and Remark 3.2, do we have $\mathcal{R}(\widetilde{T})=\mathcal{E} \mathcal{R}(T)$ in the nonAbelian case?

(4) Does Corollary 3.3 extend to the non-Abelian case: i.e. does $\mathcal{E} \mathcal{R}(T)=\mathcal{R}(\widehat{T})$, where $\widehat{T}$ is an extension of $T$ to a $G$-action on the same measure space, where $T$ is defined? 
(5) A multiple recurrence (and even recurrence) along directions can be defined in the following way. Let $T$ be a measure-preserving action of $\Gamma$ on a $\sigma$-finite measure space $(X, \mu)$ and let $p \in \mathbb{N}$. We call $T$ p-recurrent along a line $\theta \in P(\mathfrak{g})$ if, for each $\epsilon>0$ and every subset $A \subset X$ of positive measure, there is an element $\gamma \in \Gamma \backslash\left\{1_{\Gamma}\right\}$ and an element $g \in \exp (\theta)$ such that $\operatorname{dist}(\gamma, g)<\epsilon$ and $\mu\left(A \cap T_{\gamma} A \cap \cdots \cap T_{\gamma}^{p} A\right)>$ 0 . Denote by $\mathcal{R}_{p}(T)$ the set of all $\theta \in P(\mathfrak{g})$ such that $T$ is $p$-recurrent along $\theta$. Then $\mathcal{R}(T)=\mathcal{R}_{1}(T) \supset \mathcal{R}_{2}(T) \supset \cdots$ and $\bigcap_{p \geq 1} \mathcal{R}_{p}(T) \supset \mathcal{R} i(T)$. We note that all these inclusions are strict and every set $\mathcal{R}_{p}(T)$ is a $G_{\delta}$. The results obtained in this work for $\mathcal{R}(T)$ extend to $\mathcal{R}_{p}(T)$ with similar proofs for each $p$.

(6) Let $T$ be a $(C, F)$-action of $\Gamma$ associated with a sequence $\left(C_{n}, F_{n-1}\right)_{n \geq 1}$ satisfying (I)-(IV) and (4.1) from $\S 4$. Given $d>0$, we denote by $C_{n}^{\otimes d}$ and $F_{n}^{\otimes d}$ the $d$ th Cartesian power of $C_{n}$ and $F_{n}$, respectively. Then the sequence $\left(C_{n}^{\otimes d}, F_{n-1}^{\otimes d}\right)_{n \geq 1}$ of subsets in $\Gamma^{d}$ satisfies (I)-(IV) and (4.1) from $\S 4$. It is easy to see that the $(C, F)$-action $T^{\otimes d}$ of $\Gamma^{d}$ is canonically isomorphic to the $d$ th tensor product of $T$ : i.e. $T_{\left(\gamma_{1}, \ldots, \gamma_{d}\right)}^{\otimes d}=T_{\gamma_{1}} \times \cdots \times T_{\gamma_{d}}$ for all $\gamma_{1}, \ldots, \gamma_{d} \in \Gamma$. The Lie algebra $\mathfrak{g}^{d}$ of $G^{d}$ is $\mathfrak{g} \otimes \cdots \otimes \mathfrak{g}(d$ times $)$. There is a natural shiftwise action of the permutation group $\Sigma_{d}$ on $\mathfrak{g}^{d}$. This action pushes down to the projective space $P\left(\mathfrak{g}^{d}\right)$. It is easy to see that the sets $\mathcal{R}\left(T^{\otimes d}\right)$ and $\mathcal{R} i\left(T^{\otimes d}\right)$ are invariant under $\Sigma_{d}$. In the case where $G=\mathbb{R}$ and $\Gamma=\mathbb{Z}$, Theorem 5.2 can be refined in the following way: given a $\Sigma_{d}$-invariant subset $\Delta \subset P\left(\mathbb{R}^{d}\right)$ and a countable $\Sigma_{d}$-invariant subset $D$ of $\Delta$, there is a rank-one free infinite measure-preserving action $T$ of $\mathbb{Z}$ such that $D \subset \mathcal{R}\left(T^{\otimes d}\right) \subset \Delta$. In particular, each countable $\Sigma_{d}$-invariant $G_{\delta}$-subset $D$ of $P\left(\mathbb{R}^{d}\right)$ is realizable as $\mathcal{R}\left(T^{\otimes d}\right)$ for some rank-one free action $T$ of $\mathbb{Z}$. This generalizes and refines partly $\dagger$ one of the main results from the recent paper by Adams and Silva [AdSi]: for each $\Sigma_{2}$-invariant subset $D$ of rational directions, there is a rank-one action $T$ of $\mathbb{Z}$ such that $D$ is the intersection of $\mathcal{R}\left(T^{\otimes 2}\right)$ with the set of all rational directions in $\mathbb{R}^{2}$. We also note that the $\mathbb{Z}^{d}$-action $T$, constructed in Theorem 5.3, has the form $T=S^{\otimes d}$ for a $(C, F)$-action $S$ of $\mathbb{Z}$.

(7) The theory of directional recurrence can be generalized in a natural way from infinite measure-preserving $\Gamma$-actions to non-singular $\Gamma$-actions.

Acknowledgements. I thank T. Meyerovitch and E. Roy for useful discussions concerning the entropy of Poisson suspensions. Part of this work was done during a visit by the author to Max Planck Institute for Mathematics, Bonn, in July 2014. The author thanks MPIM for their support and hospitality.

\section{REFERENCES}

[Aa] J. Aaronson. Introduction to Infinite Ergodic Theory (Mathematical Surveys and Monographs, 50). American Mathematical Society, Providence, RI, 1997.

[AdSi] T. M. Adams and C. E. Silva. On infinite transformations with maximal control of ergodic two-fold product powers. Israel J. Math. 209 (2015), 929-948.

$\dagger$ This refinement is partial because we consider only the recurrence set while Adams and Silva studied, simultaneously, the set of rational ergodic directions for $T^{\otimes 2}$. 
[Au-Ha] L. Auslander, L. W. Green and F. Hahn. Flows on Homogeneous Spaces. Princeton University Press, Princeton, NJ, 1963.

[Da1] A. I. Danilenko. ( $C, F)$-Actions in Ergodic Theory (Progress in Mathematics, 265). Birkhäuser, Basel, 2008, pp. 325-351.

[Da3] A. I. Danilenko. Actions of finite rank: weak rational ergodicity and partial rigidity. Ergod. Th. \& Dynam. Sys., to appear.

[DaLe] A. I. Danilenko and M. Lemańczyk. Odometer actions of the Heisenberg group. J. Anal. Math., to appear.

[DaSi] A. I. Danilenko and C. E. Silva. Ergodic Theory: Nonsingular Transformations (Encyclopedia of Complexity and Systems Science). Springer, New York, 2009, pp. 3055-3083.

[Fel] J. Feldman. A ratio ergodic theorem for commuting, conservative, invertible transformations with quasi-invariant measure summed over symmetric hypercubes. Ergod. Th. \& Dynam. Sys. 27 (2007), 1135-1142.

[FeKa] S. Ferenczi and B. Kamiński. Zero entropy and directional Bernoullicity of a Gaussian $\mathbb{Z}^{2}$-action. Proc. Amer. Math. Soc. 123 (1995), 3079-3083.

[Ja-Ru] E. Janvresse, T. Meyerovitch, E. Roy and T. de la Rue. Poisson suspensions and entropy for infinite transformations. Trans. Amer. Math. Soc. 362 (2010), 3069-3094.

[JoSa] A. S. A. Johnson and A. A. Şahin. Directional recurrence for infinite measure preserving $\mathbb{Z}^{d}$-actions. Ergod. Th. \& Dynam. Sys. 35 (2015), 2138-2150.

[Ma] G. Mackey. Induced representations of locally compact groups. I. Ann. of Math. (2) 55 (1952), 101-139.

[Mal] A. I. Malcev. On a class of homogeneous spaces. Izv. Akad. Nauk. SSSR Ser. Mat. 13 (1949), 9-32 (in Russian).

[Mi] J. Milnor. On the entropy geometry of cellular automata. Complex Systems 2 (1988), 357-385.

[Ne] Y. Neretin. Categories of Symmetries and Infinite-Dimensional Groups (London Mathematical Society Monographs. New Series, 16). Oxford Science Publications, The Clarendon Press, Oxford University Press, New York, 1996.

[Pa] K. K. Park. On directional entropy functions. Israel J. Math. 113 (1999), 243-267.

[Ro] E. Roy. Poisson suspensions and infinite ergodic theory. Ergod. Th. \& Dynam. Sys. 29 (2009), 667-683.

[Zi] R. J. Zimmer. Induced and amenable ergodic actions of Lie groups. Ann. Sci. Éc. Norm. Supér. 11 (1978), 407-428. 